\title{
When, Where and How to Estimate Persistent and Transient Efficiency in Stochastic Frontier Panel Data Models
}

\author{
Oleg Badunenko* \\ Faculty of Management, Economics and Social Sciences \\ University of Cologne \\ Subal C. Kumbhakar ${ }^{\dagger}$ \\ Department of Economics \\ State University of New York at Binghamton
}

\begin{abstract}
In this paper we examine robustness of a recently developed panel data stochastic frontier model that allows for both persistent and transient (also known as long-run and short-run or time-invariant and time-varying) inefficiency along with random firm-effects (heterogeneity) and noise. We address some concerns that the practitioners might have about this model. First, given that there are two random time-invariant components (persistent inefficiency and firm-effects) the concern is whether the model can accurately identify them, and if so how precisely can the model estimate them? Second, there are two time-varying random components (transient inefficiency and noise), and the concern is whether the model can separate noise from transient inefficiency, and if so how precisely can the model estimate transient inefficiency? Third, how well are persistent and transient inefficiency estimated under different scenarios, viz., under different configurations of the variance parameters of the four random components? Given that the model is quite complex, relatively new and becoming quite popular in the panel efficiency literature, we feel that there is need for a detailed simulation study to examine when, where and how one can use this model with confidence to estimate persistent and transient inefficiency.
\end{abstract}

JEL Classification: C23, D24

Keywords: (D) Production/Cost Function, Heterogeneity, Inefficiency, Closed-Skew Normal Distribution, Simulated Maximum Likelihood, Simulations

\footnotetext{
${ }^{*}$ Corresponding author, Oleg Badunenko, Faculty of Management, Economics and Social Sciences, University of Cologne, Albertus-Magnus-Platz, 50923, Cologne, Germany. Phone: +49 2214701285 . Fax: +49 221470 2313, E-mail: obadunen@uni-koeln.de.

${ }^{\dagger}$ Subal C. Kumbhakar, Department of Economics, State University of New York, Binghamton, NY 13902-6000, USA. Phone: 607-777-4762, Fax: 607-777-2681, E-mail: kkar@binghamton.edu.
} 


\section{Introduction}

Now-a-days panel data models are extensively used in almost every area of microeconometric applications. Efficiency modeling is not an exception to this trend. In efficiency estimation panel models are increasingly used by the academicians (since the early 1980s) as well as in regulatory agencies in more recent years. For example, the regulators in the UK not only use panel data to increase number of observations and precision of the parameters in cases where cross-sectional units are small, many of them also use state-of-the-art efficiency models. Office of the Rail and Road in the UK use panel data models to examine both persistent and transient inefficiency. Other UK regulatory agencies (Ofwat, Ofgem, Royal Mail, etc.) are also interested in separating persistent inefficiency from firm-heterogeneity (often related to special factors), in addition to examining time-varying inefficiency in both price setting and merger cases.

In examining efficiency regulators often give special allowance to some companies because of their special production conditions, locations, etc. The allowance is somewhat ad hoc because it is not estimated from any formal economic/econometric model and therefore there is no way of knowing whether it captures firm heterogeneity or persistent inefficiency or both. It is perhaps better to decide on the special factor allowance from a formal model so that it becomes transparent to all the firms that are being regulated. Since the regulators all over the world use carrots and stick principle, it is also desirable for both the regulators and the firms being regulated to know whether carrots and sticks are equally applicable to persistent and transient inefficiency. For this, one needs to know where and when persistent inefficiency can be accurately estimated. The other important issue in regulatory cases is whether the regulated firms are improving their efficiency over time to attain the benchmark (catch-up effect). In doing so one has to estimate time-varying (transient) inefficiency and again it is important to know that the transient component is estimated accurately, so that no undue burden is placed on the firms being regulated in achieving a target that is not estimated accurately.

To address these issues the stochastic frontier model that was introduced recently (Colombi et al. (2014), Kumbhakar et al. (2014), Tsionas and Kumbhakar (2014)) has four components, viz., persistent and transient inefficiency, random firm-effects (firm heterogeneity) and noise. Because of the complexity of the model different estimation methods are proposed. For example, Colombi et al. (2014) used a full maximum likelihood method, Kumbhakar et al. (2014) used a multi-step approach, Tsionas and Kumbhakar (2014) used a Bayesian approach and finally Filippini and Greene (2016) used the simulated maximum likelihood (SML) approach. In our simulations we use the SML method to estimate the models designed to address the concern 'when, where and how one can use this model with confidence to estimate persistent and transient inefficiency'.

Our results show that the reliability of estimation of persistent and transient technical efficiency critically depends on three estimated parameters, viz., (i) the ratio of the variance parameter in persistent technical inefficiency to the variance parameter in random effects, (ii) 
the ratio of the variance parameter in transient technical inefficiency to the variance parameter in noise, and (iii) the ratio of the the variance parameter in persistent technical inefficiency to the the variance parameter in transient technical inefficiency. Specifically, the estimator does a good job estimating persistent technical efficiency (transient technical efficiency) for relatively large values of the first (second) ratio. The third ratio plays a corrective role in the accuracy of the estimates.

It is important to note that in nearly all the cases the estimator can estimate either persistent or transient technical efficiency reliably. Only in the first and second cases when the variance parameters are relatively high (high variation of persistent technical inefficiency relative to random effects and high variation of transient technical inefficiency relative to noise) the estimator provides accurate technical efficiency estimates of both persistent and transient technical efficiency. If both ratios are relatively low, the estimator cannot be trusted for estimating either type of technical efficiency.

It is worth emphasizing that in practice the ratios are not known. Using four empirical examples, we provide a simple guide on how to judge the reliability of the obtained estimates. We show that the estimator is not consistent with some of the data sets which are used in efficiency analysis using other restrictive models.

The rest of the paper is organized as follows: Section 2 provides a description of the estimator. The third section gives the details of the Monte Carlo study as well as the results of the simulations. In Section 4 we apply the estimator on eight data sets to see how it performs in practice and relate the empirical results to those from simulations. The last section concludes.

\section{Stochastic Frontier Model for Panel Data}

The stochastic frontier (SF) model originally proposed by Aigner et al. (1977) and Meeusen and van den Broeck (1977) has traveled a long way since its inception. The panel version of the standard 1977 SF model (without any amendments) can be written as

$$
\begin{aligned}
y_{i t} & =\boldsymbol{x}_{i t} \boldsymbol{\beta}+v_{i t}-\mathbf{p} \cdot u_{i t} \\
& =\boldsymbol{x}_{i t} \boldsymbol{\beta}+\epsilon_{i t},
\end{aligned}
$$

where $i=1, \cdots, n$ denotes observation and $t=1, \cdots, T_{i}$ denotes time period. In a SF frontier model, the outcome variable $y_{i t}$ is the logarithm of output, $\boldsymbol{x}_{i t}$ is the row vector of a constant, $\log$ arithms of the input variables and possibly other observed covariates that include environmental variables that are not primary inputs but nonetheless affect output. $\mathrm{p}$ is a known parameter to distinguish between production and cost function models, viz.,

$$
p= \begin{cases}1 & \text { for a stochastic production frontier model } \\ -1 & \text { for a stochastic cost frontier model }\end{cases}
$$


The random noise term $v_{i t}$ is assumed to be i.i.d. normal with zero mean and variance $\sigma_{v}^{2}$. Similarly, $u_{i t} \geq 0$ is the time-varying technical inefficiency term which is assumed to be i.i.d. as half normal, that is, $u_{i t}=\left|U_{i t}\right|$, where $U_{i t}$ is $i . i . d$. normal with zero mean and variance $\sigma_{u}^{2}$. Note that this model is simply a pooled cross-sectional model with the additional subscript $t$ which is redundant because of the i.i.d. nature of both noise and inefficiency.

In several papers, Kumbhakar (1991), Kumbhakar and Heshmati (1995), Kumbhakar and Hjalmarsson $(1993,1995)$ interpreted $u_{i t} \geq 0$ as time-varying technical inefficiency and added an extra component $u_{0 i} \geq 0$ to represent persistent inefficiency (in addition to the noise term $\left.v_{i t}\right)$. In other words, in the models used by Kumbhakar and coauthors in the 1990s inefficiency is decomposed into two parts: persistent and time-varying, $u_{0 i}$ and $u_{i t}$. The persistent component is consistent with the models used in the 1980s (Pitt and Lee 1981, Schmidt and Sickles 1984, Kumbhakar 1987, Battese and Coelli 1988), whereas the time-varying component is consistent with the models developed in the 1990s (Kumbhakar 1990, Cornwell et al. 1990, Battese and Coelli 1992) in which $u_{i t}$ is allowed to vary over time either by assuming it to be i.i.d. over $i$ and $t$ or making its mean/variance parameter a function of other exogenous variables varying over $i$ and $t$. Quantifying the magnitude of persistent inefficiency is important, especially in short panels, because it reflects the effects of inputs like management (Mundlak 1961) as well as other unobserved factors that vary across firms but not over time. Thus, unless there is a change in something that affects the management practices at the firm level (such as changes in ownership or new government regulations), it is unlikely that persistent inefficiency will change. Alternatively, time varying efficiency can change over time without operational changes in the firm.

There is, however, a philosophical question about interpreting $u_{0 i}$ as persistent inefficiency. Should one view it as the persistent inefficiency as in Kumbhakar (1991), Kumbhakar and Heshmati (1995), Kumbhakar and Hjalmarsson (1993, 1995) or as firm-heterogeneity that captures the effects of (unobserved) time-invariant covariates that has nothing to do with inefficiency? Mester (1997) for example argues that the estimates of efficiencies in stochastic frontier model are biased if heterogeneity is ignored. If one treats $u_{0 i}, i=1, \cdots, N$ as a random variable representing firm heterogeneity and is uncorrelated with $\boldsymbol{x}_{i t}$ then the above three-component model becomes the 'true random-effects' (TRE) model (Greene 2005). ${ }^{1}$ Bos et al. (2009) account for sample heterogeneity by shifting the underlying technology. Lee (2010) also estimate different frontiers, but this is not the same as accounting for unobserved heterogeneity. Thus, the difference between the TRE and the models proposed by Kumbhakar and coauthors mentioned above is in the interpretation of the 'time-invariant' term, $u_{0 i}$, i.e., whether it is persistent inefficiency or firm-effects.

\footnotetext{
${ }^{1}$ Kumbhakar and Wang (2005) developed a similar model in which the firm-effects are treated as fixed but they modeled time-varying inefficiency in more general terms by allowing factors that can affect it.
} 


\subsection{Model that Accounts for Heterogeneity and Persistent Inefficiency}

Recently Colombi et al. (2014), Kumbhakar et al. (2014), Tsionas and Kumbhakar (2014) introduced a model that split the error term into four components. The first component captures firms' latent heterogeneity (Greene 2005) and the second component captures long-run (persistent) inefficiency as in Kumbhakar and Hjalmarsson (1993), Kumbhakar and Heshmati (1995), Kumbhakar and Hjalmarsson (1995), both of which are time-invariant. The third component captures short-run/transient/time-varying inefficiency while the last component captures random shocks. Both the third and fourth components are observation-specific (i.e., vary across firms and over time). ${ }^{2}$ The model is formally expressed as

$$
\begin{aligned}
y_{i t} & =\boldsymbol{x}_{i t} \boldsymbol{\beta}+v_{0 i}-\mathbf{p} \cdot u_{0 i}+v_{i t}-\mathbf{p} \cdot u_{i t} \\
& =\boldsymbol{x}_{i t} \boldsymbol{\beta}+\epsilon_{0 i}+\epsilon_{i t}
\end{aligned}
$$

where $u_{0 i}>0$ and $u_{i t}>0$ represent long-run and short-run inefficiency, respectively, while $v_{0 i}$ captures latent firm heterogeneity and $v_{i t}$ is the classical random noise. We define, $\epsilon_{i t}=v_{i t}-\mathrm{p} \cdot u_{i t}$ and $\epsilon_{0 i}=v_{0 i}-\mathrm{p} \cdot u_{0 i}$ which decomposes the error term into two 'composed error' terms (both of which contain an inefficiency and a noise term). This decomposition will be useful later when we discuss estimation of the model.

The above decomposition might be desirable for policy purposes, especially in regulated industries. Since $u_{0 i}$ does not change over time, if a regulator wants to improve efficiency, then some fundamental change in management or policy needs to occur. In regulated industry all the firms might be operating under excess capacity which might be reflected in high values of $u_{0 i}$ but so long as $u_{0 i}$ are similar among firms, relative persistent inefficiency among firms will be small. In such a case ranking of firms based on relative values of $u_{0 i}$ will be quite similar and the regulator cannot punish some firms because all firms have high values of $u_{0 i}$. However, the estimates of $u_{0 i}$ provide useful information about the firms in the industry because high values of $u_{0 i}$ are indicators of non-competitive market condition. This is because in a competitive market there is no persistent inefficiency, i.e., persistently inefficient firms will go out of business. The short-run inefficiency can be adjusted over time without a major policy change. Thus, for example, if the short-run inefficiency component for a firm is relatively large in a particular year then it may be argued that inefficiency is caused by something which is unlikely to be repeated in the next year. On the other hand, if the persistent inefficiency component is large for a firm, then it is expected to operate with a relatively high level of inefficiency over time, unless some changes in policy and/or management take place. Thus, a high value of $u_{0 i}$ is of more concern from a long term point of view because of its persistent nature than is a high value of $u_{i t}$.

\footnotetext{
${ }^{2}$ Yip et al. (2011) argue that heterogeneity needs to be separated from inefficiency, but they still estimate only time-varying inefficiency similar to Greene (2005).
} 


\subsubsection{Full Maximum Likelihood Method}

To obtain a tractable likelihood function, Colombi et al. (2014) draw results from skew normal and closed skew normal (CSN) distributions. Assuming $v_{i t}$ is i.i.d. normal and $u_{i t}$ is i.i.d. half normal, $\epsilon_{i t}$ in (3b) has a skew normal distribution. Using the same argument $\epsilon_{0 i}$ in (3b) has a skew normal distribution when $v_{0 i}$ is i.i.d. normal and $u_{0 i}$ is i.i.d. half normal. Thus, the composed error term $\epsilon_{0 i}+\epsilon_{i t}$ in (3a) has a CSN distribution (being the sum of two independent skew normal distributions) which has a well defined $p d f$ that is used to define the log-likelihood function maximization of which gives MLE of all the parameters. This is the estimation strategy used by Colombi et al. (2014).

The model in (3a) can be rewritten in a compact form, viz.,

$$
\boldsymbol{y}_{i}=\boldsymbol{x}_{i} \boldsymbol{\beta}+\mathbf{1}_{T_{i}} v_{0 i}+\boldsymbol{A} \boldsymbol{u}_{i}+\boldsymbol{v}_{i}
$$

where $\boldsymbol{u}_{i}=\left(u_{0 i}, u_{i 1}, \ldots, u_{i T_{i}}\right)^{\prime}$ and $\boldsymbol{v}_{i}=\left(v_{i 1}, \ldots, v_{i T_{i}}\right)^{\prime}$ are vectors of length $T_{i}, \boldsymbol{A}=-\mathrm{p} \times$ $\left[\begin{array}{ll}\mathbf{1}_{T_{i}} & \boldsymbol{I}_{T_{i}}\end{array}\right]$ is a matrix of dimension $T_{i} \times\left(T_{i}+1\right)$, where $\mathbf{1}_{T_{i}}$ is the column vector of length $T_{i}$, and $\boldsymbol{I}_{T_{i}}$ is the identity matrix of dimension $T_{i}$. Colombi et al. (2014) derive the joint density function of the composed error term $\boldsymbol{\epsilon}_{i}=\mathbf{1}_{T_{i}} v_{0 i}+\boldsymbol{A} \boldsymbol{u}_{i}+\boldsymbol{v}_{i}$ based on the result that $\boldsymbol{\epsilon}_{i}$ follows a CSN distribution. The resulting panel-level log-likelihood function of the four component model is (see Colombi et al. (2014) for details):

$$
\begin{aligned}
\log L_{i}\left(\boldsymbol{\beta}, \sigma_{v}, \boldsymbol{\sigma}_{u}, \sigma_{v_{0}}, \sigma_{u_{0}}\right) & =\left(T_{i}+1\right) \log 2+\log \phi_{T_{i}}\left(\boldsymbol{\epsilon}_{i}, \mathbf{0}, \boldsymbol{\Sigma}+\boldsymbol{A} \boldsymbol{V} \boldsymbol{A}^{\prime}\right) \\
& +\log \bar{\Phi}_{T_{i}+1}\left(\boldsymbol{R} \boldsymbol{\epsilon}_{i}, \boldsymbol{\Lambda}\right)
\end{aligned}
$$

where $\boldsymbol{\epsilon}_{i}=\boldsymbol{y}_{i}-\boldsymbol{x}_{i} \boldsymbol{\beta}$, the diagonal elements of $\boldsymbol{V}$ are $\left(\sigma_{u_{0}}, \boldsymbol{\sigma}_{u}\right),{ }^{3} \boldsymbol{\Sigma}=\sigma_{v}^{2} \boldsymbol{I}_{T_{i}}+\sigma_{v_{0}}^{2} \mathbf{1}_{T_{i}} \mathbf{1}_{T_{i}}^{\prime}, \boldsymbol{\Lambda}=$ $\boldsymbol{V}-\boldsymbol{V} \boldsymbol{A}^{\prime}\left(\boldsymbol{\Sigma}+\boldsymbol{A} \boldsymbol{V} \boldsymbol{A}^{\prime}\right)^{-1} \boldsymbol{A} \boldsymbol{V}=\left(\boldsymbol{V}^{-1}+\boldsymbol{A}^{\prime} \boldsymbol{\Sigma}^{-1} \boldsymbol{A}\right)^{-1}, \boldsymbol{R}=\boldsymbol{V} \boldsymbol{A}^{\prime}\left(\boldsymbol{\Sigma}+\boldsymbol{A} \boldsymbol{V} \boldsymbol{A}^{\prime}\right)^{-1}=\boldsymbol{\Lambda} \boldsymbol{A}^{\prime} \boldsymbol{\Sigma}^{-1}$, $\phi_{q}(x, \boldsymbol{\mu}, \boldsymbol{\Omega})$ is the density function of a $q$-dimensional normal variable with expected value $\boldsymbol{\mu}$ and variance $\boldsymbol{\Omega}$, and $\bar{\Phi}_{q}(\boldsymbol{\mu}, \boldsymbol{\Omega})$ is the probability that a $q$-variate normal variable of expected value $\boldsymbol{\mu}$ and variance $\boldsymbol{\Omega}$ belongs to the positive orthant.

An additional interesting result in Colombi et al. (2014) is that on can predict technical/cost efficiencies as follows:

$$
E\left(\exp \left(\boldsymbol{t}^{\prime} \boldsymbol{u}_{i}\right) \mid \boldsymbol{y}_{i}\right)=\frac{\bar{\Phi}_{T_{i}+1}\left(\boldsymbol{R} \boldsymbol{\epsilon}_{i}+\boldsymbol{\Lambda} \boldsymbol{t}, \boldsymbol{\Lambda}\right)}{\bar{\Phi}_{T_{i}+1}\left(\boldsymbol{R} \boldsymbol{\epsilon}_{i}, \boldsymbol{\Lambda}\right)} \times \exp \left(\boldsymbol{t}^{\prime} \boldsymbol{R} \boldsymbol{\epsilon}_{i}+0.5 \boldsymbol{t}^{\prime} \boldsymbol{\Lambda} \boldsymbol{t}\right),
$$

where $-\boldsymbol{t}$ is a row of the identity matrix of dimension $\left(T_{i}+1\right)$. If $-\boldsymbol{t}$ is the $\tau$-th row, Eq (6) provides the conditional expected value of the $\tau$-th component of the technical/cost efficiency vector $\exp \left(-\boldsymbol{u}_{i}\right)$. In particular, for $\tau=1$, we get the conditional expected value of the persistent technical/cost efficiency. For firm $i$ at time $t$ the overall efficiency is obtained as a product of the persistent and transient technical efficiencies, $\mathrm{TE}_{i t}^{\text {overall }}=\mathrm{TE}_{i}^{\mathrm{persistent}} \cdot \mathrm{TE}_{i t}^{\mathrm{transient}}$. Note that

\footnotetext{
${ }^{3}$ Note that $\boldsymbol{\sigma}_{u}=\mathbf{1}_{T_{i}} \sigma_{u}$ becomes $\left(\sigma_{u 1}, \ldots, \sigma_{u T_{i}}\right)$ in heteroskedastic case.
} 
technical efficiency is defined as $\mathrm{TE}_{i}=\exp \left(-u_{0 i}\right)$ and $\mathrm{TE}_{i t}=\exp \left(-u_{i t}\right)$.

\subsubsection{A Simulated Maximum Likelihood Estimator}

Although the CSN framework gives a closed form expression of the log-likelihood function, implementing it in practice is a daunting task. Recently Filippini and Greene (2016) proposed a simulation based optimization routine which circumvents many of the challenges associated with brute force optimization in this setting. Using the insights of Butler and Moffitt (1982), Filippini and Greene (2016) note that the density in Colombi et al. (2014) can be greatly simplified by conditioning on $v_{0 i}$ and $u_{0 i}$. In this case, the conditional density is simply the product over time of $T_{i}$ univariate skew normal densities. Thus, only a single integral, as opposed to $T_{i}$ integrals needs to be evaluated. Maximization of this simulated log likelihood is not more complicated from the cross sectional case, aside from the additional parameters.

Recall that $\epsilon_{i t}$ is a skew normal variate with parameters $\lambda=\sigma_{u} / \sigma_{v}$ and $\sigma=\left(\sigma_{u}^{2}+\sigma_{v}^{2}\right)^{1 / 2}$. Similarly, $\epsilon_{0 i}$ is a skew normal variate with parameters $\lambda_{0}=\sigma_{u_{0}} / \sigma_{v_{0}}$ and $\sigma_{0}=\left(\sigma_{u_{0}}^{2}+\sigma_{v_{0}}^{2}\right)^{1 / 2}$. Thus, the conditional density of $\boldsymbol{\epsilon}_{i}=\left(\epsilon_{i 1}, \ldots, \epsilon_{i T_{i}}\right)$ is given by

$$
f\left(\epsilon_{i} \mid \epsilon_{0 i}\right)=\prod_{t=1}^{T_{i}} \frac{2}{\sigma} \phi\left(\frac{\epsilon_{i t}}{\sigma}\right) \Phi\left(\frac{-\mathrm{p} \cdot \epsilon_{i t} \lambda}{\sigma}\right) .
$$

Integrate $\epsilon_{0 i}$ (distribution of which we know) out to get the unconditional density of $\boldsymbol{\epsilon}_{i}$

$$
f\left(\boldsymbol{\epsilon}_{i}\right)=\int_{-\infty}^{\infty}\left[\prod_{t=1}^{T_{i}} \frac{2}{\sigma} \phi\left(\frac{\epsilon_{i t}}{\sigma}\right) \Phi\left(\frac{-\mathrm{p} \cdot \epsilon_{i t} \lambda}{\sigma}\right)\right] \times \frac{2}{\sigma_{0}} \phi\left(\frac{\epsilon_{0 i}}{\sigma_{0}}\right) \Phi\left(\frac{-\mathrm{p} \cdot \epsilon_{0 i} \lambda_{0}}{\sigma_{0}}\right) d \epsilon_{0 i}
$$

The log-likelihood function for the $i$-th observation of the model (3b) is therefore given by

$$
\begin{aligned}
& \log L_{i}\left(\boldsymbol{\beta}, \lambda, \sigma, \lambda_{0}, \sigma_{0}\right) \\
& =\log \left[\int_{-\infty}^{+\infty}\left(\prod_{t=1}^{T_{i}}\left\{\begin{array}{l}
\frac{2}{\sigma} \phi\left(\frac{y_{i t}-\boldsymbol{x}_{i t} \boldsymbol{\beta}-\epsilon_{0 i}}{\sigma}\right) \\
\times \Phi\left(\frac{-\mathrm{p} \cdot\left(y_{i t}-\boldsymbol{x}_{i t} \boldsymbol{\beta}-\epsilon_{0 i}\right) \lambda}{\sigma}\right)
\end{array}\right\}\right) \frac{2}{\sigma_{0}} \phi\left(\frac{\epsilon_{0 i}}{\sigma_{0}}\right) \Phi\left(\frac{-\mathrm{p} \cdot \epsilon_{0 i} \lambda_{0}}{\sigma_{0}}\right) \mathrm{d} \epsilon_{0 i}\right] \\
& =\log \left[\int_{-\infty}^{+\infty}\left(\prod_{t=1}^{T_{i}}\left\{\frac{2}{\sigma} \phi\left(\frac{\epsilon_{i t}}{\sigma}\right) \Phi\left(\frac{-\mathrm{p} \cdot \epsilon_{i t} \lambda}{\sigma}\right)\right\}\right) \times \frac{2}{\sigma_{0}} \phi\left(\frac{\epsilon_{0 i}}{\sigma_{0}}\right) \Phi\left(\frac{-\mathrm{p} \cdot \epsilon_{0 i} \lambda_{0}}{\sigma_{0}}\right) \mathrm{d} \epsilon_{0 i}\right]
\end{aligned}
$$

where $\epsilon_{i t}=y_{i t}-\boldsymbol{x}_{i t} \boldsymbol{\beta}-\left(v_{0 i}-\mathrm{p} \cdot u_{0 i}\right)$. Although, following CSN, one can derive the likelihood function in closed form, we approximate the log-likelihood function and avoid using the classical ML method which is quite complicated. We rely on the Monte-Carlo integration as a method to approximate the integral (9b). For estimation purposes we write $\epsilon_{0 i}=\sigma_{v_{0}} V_{i}-\mathrm{p} \cdot \sigma_{u_{0}}\left|U_{i}\right|$, where both $V_{i}$ and $U_{i}$ is standard normal random variable. The resulting simulated log-likelihood 
function for the $i$-th observation is

$$
\begin{aligned}
& \log L_{i}^{S}\left(\boldsymbol{\beta}, \lambda, \sigma, \sigma_{v_{0}}, \sigma_{u_{0}}\right) \\
& =\log \left[\frac{1}{R} \sum_{r=1}^{R}\left(\prod_{t=1}^{T_{i}}\left\{\begin{array}{l}
\frac{2}{\sigma} \phi\left(\frac{y_{i t}-\boldsymbol{x}_{i t} \boldsymbol{\beta}-\left(\sigma_{v_{0}} V_{i r}-\mathrm{p} \cdot \sigma_{u_{0}}\left|U_{i r}\right|\right)}{\sigma}\right) \\
\times \Phi\left(\frac{-\mathrm{p} \cdot\left[y_{i t}-\boldsymbol{x}_{i t} \boldsymbol{\beta}-\left(\sigma_{v_{0}} V_{i r}-\mathrm{p} \cdot \sigma_{u_{0}}\left|U_{i r}\right|\right)\right] \lambda}{\sigma}\right)
\end{array}\right\}\right)\right] \\
& =\log \left[\frac{1}{R} \sum_{r=1}^{R}\left(\prod_{t=1}^{T_{i}}\left\{\frac{2}{\sigma} \phi\left(\frac{\epsilon_{i t r}}{\sigma}\right) \Phi\left(\frac{-\mathrm{p} \cdot \epsilon_{i t r} \lambda}{\sigma}\right)\right\}\right)\right]
\end{aligned}
$$

where $V_{i r}$ and $U_{i r}$ are $R$ random deviates from the standard normal distribution, and $\epsilon_{i t r}=$ $y_{i t}-\boldsymbol{x}_{i t} \boldsymbol{\beta}-\left(\sigma_{v_{0}} V_{i r}-\mathrm{p} \cdot \sigma_{u_{0}}\left|U_{i r}\right|\right) . R$ is the number of draws for approximating the log-likelihood function.

After estimating $\boldsymbol{\beta}$ and the parameters of the distributions of the random components, we use the moment generating function of Colombi et al. (2014) in (6) which provide us the conditional means of $u_{0 i}, u_{i 1}, \cdots, u_{i T_{i}}$ which are, in principle, similar to the Jondrow et al. (1982) estimator.

\section{Simulations}

In this section we examine the finite sample performance of the estimator that approximates the integral in (9b) using the Monte-Carlo integration. Specifically, we first discuss the basic design of the experiment. We define the data generating processes used, the distributional assumptions on the data as well as the other parameters of the experiment. Next, we discuss the methods to compare the performance of the efficiency estimators. We take several standard measures as well as one that we propose just for this experiment. Third, we run our simulations and discuss the relative performance of the estimators under various scenarios.

\subsection{Design of the Experiment}

We conduct simulations for a production process which employs two inputs $\left(X_{1}\right.$ and $\left.X_{2}\right)$ to produce a single output $(Y)$. We consider the following Cobb-Douglas (CD) production functions $Y=\exp (a) \cdot X_{1}^{\alpha} X_{2}^{\gamma-\alpha}$. Note that the uppercase letters are used for the input and output variables in levels. We assume the output is generated via a constant returns to scale (CRS) technology $(\gamma=1)$. Further, we set $a=0.3$ and $\alpha=0.4$.

We assume that the true error term is distributed normally with mean zero and variance $\sigma_{v}^{2}$, i.e., $v_{i t} \sim N\left(0, \sigma_{v}^{2}\right)$. We further assume that the true random effects component is distributed normally with zero mean and variance $\sigma_{v_{0}}^{2}$, i.e., $v_{0 i} \sim N\left(0, \sigma_{v_{0}}^{2}\right)$. Next, we assume that the true persistent technical efficiency is $\mathrm{TE}_{i}=\exp \left(-u_{0 i}\right)$, where $u_{0 i}$ is assumed to be half-normally distributed, i.e., $u_{0 i}=\left|U_{i}\right|$ and $U_{i} \sim N\left(0, \sigma_{u_{0}}^{2}\right)$. We assume that the true transient technical efficiency is $\mathrm{TE}_{i t}=\exp \left(-u_{i t}\right)$, where $u_{i t}=\left|U_{i t}\right|$ and $U_{i t} \sim N\left(0, \sigma_{u}^{2}\right)$. We introduce the noise 
and technical inefficiency in the production process of unit $i$ as

$$
Y_{i t}=\exp (a) \cdot X_{1 i t}^{\alpha} X_{2 i t}^{\gamma-\alpha} \exp \left(v_{0 i}-u_{0 i}+v_{i t}-u_{i t}\right)
$$

To model various real-life scenarios, we have simulated 16 different combinations for the variation of the error, random effects, and two technical inefficiency terms. Table 1 lists all possible scenarios. One way to summarize the cases is in terms of ratios of the variance parameters, viz., variance parameter of the technical inefficiency component to the noise component $\left(\lambda_{0}=\right.$ $\left.\sigma_{u_{0}} / \sigma_{v_{0}}\right)$, and the ratio of the variance parameter of the transient technical efficiency to the variance of the idiosyncratic error term $\left(\lambda=\sigma_{u} / \sigma_{v}\right)$. Table 2 shows the matrix of chosen combinations when $\sigma_{v_{0}}, \sigma_{u_{0}}, \sigma_{v}$, and $\sigma_{u}$ all take values of 0.04 and 0.2 . For example, scenario s1 is where all $\sigma_{v_{0}}, \sigma_{u_{0}}, \sigma_{v}$, and $\sigma_{u}$ take the value 0.04 . In scenario s2, the true variance parameter of transient technical inefficiency component changes, $\sigma_{u}=0.2$; in s3, the true variance parameter of persistent technical inefficiency is changed: $\sigma_{u_{0}}=0.2$ etc.

Table 1: Combinations of $\lambda$ and $\sigma$ values used in the Monte Carlo simulations

\begin{tabular}{rrrrrrrr}
\hline & $\sigma_{u_{0}}$ & $\sigma_{u}$ & $\sigma_{v_{0}}$ & $\sigma_{v}$ & $\lambda_{0}$ & $\lambda$ & $\Lambda$ \\
\hline $\mathrm{s} 1$ & 0.04 & 0.04 & 0.04 & 0.04 & 1 & 1 & 1 \\
$\mathrm{~s} 2$ & 0.04 & 0.2 & 0.04 & 0.04 & 1 & 5 & 0.2 \\
$\mathrm{~s} 3$ & 0.2 & 0.04 & 0.04 & 0.04 & 5 & 1 & 5 \\
$\mathrm{~s} 4$ & 0.2 & 0.2 & 0.04 & 0.04 & 5 & 5 & 1 \\
$\mathrm{~s} 5$ & 0.04 & 0.04 & 0.04 & 0.2 & 1 & 0.2 & 1 \\
$\mathrm{~s} 6$ & 0.04 & 0.2 & 0.04 & 0.2 & 1 & 1 & 0.2 \\
$\mathrm{~s} 7$ & 0.2 & 0.04 & 0.04 & 0.2 & 5 & 0.2 & 5 \\
$\mathrm{~s} 8$ & 0.2 & 0.2 & 0.04 & 0.2 & 5 & 1 & 1 \\
$\mathrm{~s} 9$ & 0.04 & 0.04 & 0.2 & 0.04 & 0.2 & 1 & 1 \\
$\mathrm{~s} 10$ & 0.04 & 0.2 & 0.2 & 0.04 & 0.2 & 5 & 0.2 \\
$\mathrm{~s} 11$ & 0.2 & 0.04 & 0.2 & 0.04 & 1 & 1 & 5 \\
$\mathrm{~s} 12$ & 0.2 & 0.2 & 0.2 & 0.04 & 1 & 5 & 1 \\
$\mathrm{~s} 13$ & 0.04 & 0.04 & 0.2 & 0.2 & 0.2 & 0.2 & 1 \\
$\mathrm{~s} 14$ & 0.04 & 0.2 & 0.2 & 0.2 & 0.2 & 1 & 0.2 \\
$\mathrm{~s} 15$ & 0.2 & 0.04 & 0.2 & 0.2 & 1 & 0.2 & 5 \\
$\mathrm{~s} 16$ & 0.2 & 0.2 & 0.2 & 0.2 & 1 & 1 & 1 \\
\hline & & & & & & &
\end{tabular}

Table 2 summarizes the information in Table 1 in terms of the ratios of the variance parameters. The first row of the Table 2 corresponds to the cases where the variance parameter of the random effects prevails over persistent technical inefficiency. In this case, we say, the decision making units are relatively efficient in the long-term. The third row of the table represents the cases where variance of persistent technical inefficiency is relatively high compared to random effects. The first column shows the cases where short-term technical inefficiency parameter is low relative to that of the noise term. The third column is for cases where the data have relatively 
Table 2: Combinations of $\lambda_{0}$ and $\lambda$ values used in various simulation scenarios

\begin{tabular}{llll}
\hline & $\lambda=0.2$ & $\lambda=1$ & $\lambda=5$ \\
\cline { 2 - 4 }$\lambda_{0}=0.2$ & $\mathrm{~s} 13$ & $\mathrm{~s} 9, \mathrm{~s} 14$ & $\mathrm{~s} 10$ \\
$\lambda_{0}=1$ & $\mathrm{~s} 5, \mathrm{~s} 15$ & $\mathrm{~s} 1, \mathrm{~s} 6, \mathrm{~s} 11, \mathrm{~s} 16$ & $\mathrm{~s} 2, \mathrm{~s} 12$ \\
$\lambda_{0}=5$ & $\mathrm{~s} 7$ & $\mathrm{~s} 3, \mathrm{~s} 8$ & $\mathrm{~s} 4$ \\
\hline
\end{tabular}

little noise, but the units under consideration are relatively inefficient in the short-term. Thus, the upper-left cell in the table where $\lambda_{0}=0.2$ and $\lambda=0.2$ represents the case where decision making units are efficient both in long- and short-term. As we move to the right (bottom), the short- (long-) term technical inefficiency get relatively larger. Although the variance parameter of four components are equably mixed, this does not result in equably filled Table 2. Four of nine cells have only one scenario, the other four have two scenarios, and the middle cell has four scenarios. Some scenarios might seem redundant, but all these cases are needed to emphasize that the results of the experiment depend upon three ratios, viz., (i) $\lambda$, (ii) $\lambda_{0}$, and (iii) $\Lambda=\sigma_{u_{0}} / \sigma_{u}-$ not on the magnitudes of the four variance parameters.

All experiments consist of 1000 Monte Carlo trials. Within each set of experiment, we analyze three sample sizes, $n=50,100$ and 500 and $t=3,6$, and 10. For each Monte Carlo trial, we simulate a DGP by drawing observations for inputs from the following density: $f_{(b)}(X)=$ $(b-1)^{-1} \exp [\log (b)-X]$, for $0<X<\log (b) .{ }^{4}$ For $X_{1}$ we set $b=2$ and for $X_{2}$ we set $b=10$. For each of the 16 combinations of $\sigma_{v_{0}}, \sigma_{u_{0}}, \sigma_{v}$, and $\sigma_{u}$, we then compute the "observed" output observations as in Eq. (11). We thus have 16 base scenarios for the assumed production function.

\subsection{Comparative measures}

To compare the finite sample performance of our estimators we consider the following mean (over the 1000 simulations) measures

$$
\begin{aligned}
& \text { Relative } \operatorname{Bias}(\mathrm{TE})=\frac{1}{B} \sum_{b=1}^{B} \frac{1}{n t} \sum_{i=1}^{n t}\left(\frac{\widehat{\mathrm{TE}}_{i b}-\mathrm{TE}_{i b}}{\mathrm{TE}_{i b}}\right) \\
& \operatorname{Upward} \operatorname{Bias}(\mathrm{TE})=\frac{1}{B} \sum_{b=1}^{B} \frac{1}{n t} \sum_{i=1}^{n t} 1 \widehat{\mathrm{TE}}_{i b}>\mathrm{TE}_{i b} \\
& \text { Pearson Correlation Coefficient }=\frac{1}{B} \sum_{b=1}^{B} \frac{\sum_{i=1}^{n t}\left(\widehat{\mathrm{TE}}_{i b}-\widehat{\mathrm{TE}}_{b}\right)\left(\mathrm{TE}_{i b}-\overline{\mathrm{TE}}_{b}\right)}{\sqrt{\sum_{i=1}^{n t}\left(\widehat{\mathrm{TE}}_{i b}-\widehat{\mathrm{TE}}_{b}\right)^{2}} \sqrt{\sum_{i=1}^{n t}\left(\mathrm{TE}_{i b}-\overline{\mathrm{TE}}_{b}\right)^{2}}}
\end{aligned}
$$

\footnotetext{
${ }^{4}$ The deviates from the $f_{(b)}$ are drawn as $\log (b)-\log (U(1, b))$.
} 
For persistent technical efficiency, $n t$ changes to $n .1_{D}$ denotes the indicator function that the event $\boldsymbol{D}$ is true, $\widehat{\mathrm{TE}}_{i}$ is the estimate of technical efficiency of unit $i$ in a given Monte Carlo replication and $\mathrm{TE}_{i}$ is the true efficiency score (determined by the DGP). In addition to the mean bias, we also consider an alternative approach to compare across estimation methods. Specifically, we give an estimate of upward bias. The upward bias measure shows the percentage of points for which technical efficiency has been overestimated. The measure checks whether an estimate is larger than the true value. If so, the indicator function returns a value of 1 . If not, then a zero value is given. The number of estimates above the true value is then divided by the sample size to give the percentage of estimates which were overestimated. Values near 0.5 are ideal for this measure. Anything in excess of 0.5 suggests an upward bias and anything less than 0.5 suggests downward bias.

We are also interested in comparing the density estimates of technical efficiency across the Monte Carlo draws. Looking at only the mean (over the simulations) may conceal interesting results. Specifically, for each draw, we sort the data by the relative value of the true efficiency. We are interested in comparing the true distribution of technical efficiency for a percentile across all Monte Carlo simulations. We therefore report mean of the relative deviation for the $\alpha$-percentile $(\alpha n)$, the mean $(n / 2)$ and the $(1-\alpha)$-percentile $((1-\alpha) n)$ of the efficiency scores. ${ }^{5}$ These show us how well each of the estimators perform at estimating particular portions of our sample. For example, if interest lies in estimating the benchmark firms, then we would be interested in knowing which estimator does the best job at estimating the $(1-\alpha)$-percentile of the efficiency distribution.

We note that the results include sampling variation apart from what we would find for individual, fixed points that do not change from one trial to the next. Put differently, the $\alpha$-percentile of firms in trial 1 is likely to be different from the $\alpha$-percentile of firms in trial 2 , and so on. An alternative approach could be to focus on one or perhaps a small set of fixed points in the input-output space that are held constant over Monte Carlo trials. We have conducted limited simulations which show that these two approaches lead to qualitatively similar results and these results are available upon request. We note that most studies surrounding efficiency scores essentially look at average behavior. We uncover different findings depending upon which percentile is being estimated. In other words, the relative performance of our estimators are heterogeneous across the sample.

\subsection{Simulation results}

Tables 3-5 and 8-10 show the performance measures of the technical efficiency estimates for various values of $\lambda_{0}$. The GTRE model performs differently depending not only on the "amount" of persistent and transient technical inefficiency, but also on the relationship between persistent and transient technical inefficiency. For different combinations of $\lambda_{0}=\sigma_{u_{0}} / \sigma_{v_{0}}, \lambda=\sigma_{u} / \sigma_{v}$,

\footnotetext{
${ }^{5}$ Mean relative deviation is defined as $\frac{1}{n t} \sum_{i=1}^{n t}\left(\frac{\widehat{\mathrm{TE}}_{i}^{\alpha-\text { percentile }}-\mathrm{TE}_{i}^{\alpha-\text { percentile }}}{\mathrm{TE}_{i}^{\alpha-\text { percentile }}}\right)$.
} 
and $\Lambda=\sigma_{u_{0}} / \sigma_{u}$ we discuss statistical properties of persistent, transient, and overall technical efficiency estimates separately. We first look at the 'average' performance of the estimator (Tables 3-5) and then present the goodness of the estimator at various cut-offs of the distribution (Tables 8-10).

\subsubsection{Estimation of technical efficiency}

We split discussion of the results into three cases, determined by the relationship between $\sigma_{u_{0}}$ and $\sigma_{v_{0}}$, viz., $\lambda_{0}$. We first focus on the case when $\sigma_{u_{0}}$ is low compared to $\sigma_{v_{0}}$, that is, when persistent technical inefficiency is almost nonexistent. The results appear in Table 3 . It is worthwhile to discuss the results by relative importance of the transient technical inefficiency $(\lambda)$ and the relationship between persistent and transient technical inefficiency $(\Lambda)$.

Table 3: Finite Sample Performance of the Technical Efficiency Estimates. $\sigma_{u_{0}}$ is lower than $\sigma_{v_{0}}$, i.e., $\lambda_{0}=0.2$.

\begin{tabular}{|c|c|c|c|c|c|c|c|c|c|c|}
\hline \multirow[b]{2}{*}{$n$} & \multirow[b]{2}{*}{$t$} & \multicolumn{3}{|c|}{ Persistent TE } & \multicolumn{3}{|c|}{ Transient TE } & \multicolumn{3}{|c|}{ Overall TE } \\
\hline & & R Bias & U Bias & Corr & R Bias & U Bias & Corr & R Bias & U Bias & Corr \\
\hline & & \multicolumn{9}{|c|}{ s13: $\lambda_{0}=0.2, \lambda=0.2, \Lambda=1\left(\sigma_{u_{0}}=0.04 \sigma_{u}=0.04, \sigma_{v_{0}}=0.2, \sigma_{v}=0.2\right)$} \\
\hline 50 & 3 & $-2.1 \mathrm{e}-2$ & 0.51 & 0.09 & $-4.1 \mathrm{e}-2$ & 0.48 & 0.10 & $-6.1 \mathrm{e}-2$ & 0.34 & 0.10 \\
\hline 100 & 3 & $-1.7 \mathrm{e}-2$ & 0.53 & 0.10 & $-3.2 \mathrm{e}-2$ & 0.49 & 0.10 & $-4.9 \mathrm{e}-2$ & 0.36 & 0.10 \\
\hline 500 & 3 & $-9.1 \mathrm{e}-3$ & 0.55 & 0.10 & $-2.0 \mathrm{e}-2$ & 0.49 & 0.10 & $-2.9 \mathrm{e}-2$ & 0.39 & 0.10 \\
\hline 50 & 6 & $-1.4 \mathrm{e}-2$ & 0.52 & 0.10 & $-2.8 \mathrm{e}-2$ & 0.51 & 0.11 & $-4.2 \mathrm{e}-2$ & 0.38 & 0.09 \\
\hline 100 & 6 & $-1.2 \mathrm{e}-2$ & 0.54 & 0.11 & $-2.2 \mathrm{e}-2$ & 0.51 & 0.11 & $-3.3 e-2$ & 0.40 & 0.10 \\
\hline 500 & 6 & $1.2 \mathrm{e}-3$ & 0.62 & 0.11 & $-1.1 \mathrm{e}-2$ & 0.51 & 0.11 & $-1.0 \mathrm{e}-2$ & 0.46 & 0.09 \\
\hline 50 & 10 & $-1.1 \mathrm{e}-2$ & 0.51 & 0.11 & $-2.3 \mathrm{e}-2$ & 0.50 & 0.11 & $-3.4 \mathrm{e}-2$ & 0.39 & 0.09 \\
\hline 100 & 10 & $-7.7 \mathrm{e}-3$ & 0.53 & 0.11 & $-2.0 \mathrm{e}-2$ & 0.49 & 0.11 & $-2.7 \mathrm{e}-2$ & 0.39 & 0.09 \\
\hline \multirow[t]{2}{*}{500} & 10 & $3.8 \mathrm{e}-3$ & 0.62 & 0.11 & $-9.1 \mathrm{e}-3$ & 0.50 & 0.11 & $-5.2 \mathrm{e}-3$ & 0.48 & 0.09 \\
\hline & & \multicolumn{9}{|c|}{$\mathrm{s} 14: \lambda_{0}=0.2, \lambda=1, \Lambda=0.2\left(\sigma_{u_{0}}=0.04 \sigma_{u}=0.2, \sigma_{v_{0}}=0.2, \sigma_{v}=0.2\right)$} \\
\hline 50 & 3 & $-2.6 \mathrm{e}-2$ & 0.52 & 0.09 & $5.0 \mathrm{e}-2$ & 0.60 & 0.45 & $2.3 \mathrm{e}-2$ & 0.53 & 0.39 \\
\hline 100 & 3 & $-2.2 \mathrm{e}-2$ & 0.52 & 0.10 & $5.0 \mathrm{e}-2$ & 0.59 & 0.46 & $2.7 \mathrm{e}-2$ & 0.53 & 0.40 \\
\hline 500 & 3 & $-1.2 \mathrm{e}-2$ & 0.55 & 0.10 & $2.9 \mathrm{e}-2$ & 0.52 & 0.46 & $1.7 \mathrm{e}-2$ & 0.49 & 0.43 \\
\hline 50 & 6 & $-1.7 \mathrm{e}-2$ & 0.52 & 0.11 & $4.6 \mathrm{e}-2$ & 0.57 & 0.48 & $2.8 \mathrm{e}-2$ & 0.53 & 0.41 \\
\hline 100 & 6 & $-1.2 \mathrm{e}-2$ & 0.54 & 0.10 & $3.3 \mathrm{e}-2$ & 0.53 & 0.49 & $2.0 \mathrm{e}-2$ & 0.50 & 0.43 \\
\hline 500 & 6 & $-4.7 \mathrm{e}-3$ & 0.58 & 0.11 & $1.5 \mathrm{e}-2$ & 0.47 & 0.49 & $9.9 \mathrm{e}-3$ & 0.47 & 0.47 \\
\hline 50 & 10 & $-1.4 \mathrm{e}-2$ & 0.51 & 0.11 & $3.3 \mathrm{e}-2$ & 0.53 & 0.50 & $1.8 \mathrm{e}-2$ & 0.50 & 0.43 \\
\hline 100 & 10 & $-1.1 \mathrm{e}-2$ & 0.52 & 0.11 & $2.0 \mathrm{e}-2$ & 0.49 & 0.50 & $8.8 \mathrm{e}-3$ & 0.47 & 0.46 \\
\hline \multirow[t]{2}{*}{500} & 10 & $6.2 \mathrm{e}-5$ & 0.59 & 0.11 & $1.0 \mathrm{e}-2$ & 0.46 & 0.50 & $1.0 \mathrm{e}-2$ & 0.47 & 0.49 \\
\hline & & \multicolumn{9}{|c|}{$\mathrm{s} 9: \lambda_{0}=0.2, \lambda=1, \Lambda=1\left(\sigma_{u_{0}}=0.04 \sigma_{u}=0.04, \sigma_{v_{0}}=0.2, \sigma_{v}=0.04\right)$} \\
\hline 50 & 3 & $-5.8 \mathrm{e}-3$ & 0.42 & 0.12 & $9.0 \mathrm{e}-3$ & 0.62 & 0.42 & $3.1 \mathrm{e}-3$ & 0.52 & 0.24 \\
\hline 100 & 3 & $-1.5 \mathrm{e}-3$ & 0.48 & 0.12 & $7.2 \mathrm{e}-3$ & 0.58 & 0.43 & $5.6 \mathrm{e}-3$ & 0.54 & 0.25 \\
\hline
\end{tabular}


Table 3 (Continued)

\begin{tabular}{|c|c|c|c|c|c|c|c|c|c|c|}
\hline \multirow[b]{2}{*}{$n$} & \multirow[b]{2}{*}{$t$} & \multicolumn{3}{|c|}{ Persistent TE } & \multicolumn{3}{|c|}{ Transient TE } & \multicolumn{3}{|c|}{ Overall TE } \\
\hline & & R Bias & U Bias & Corr & R Bias & U Bias & Corr & R Bias & U Bias & Corr \\
\hline 500 & 3 & $1.2 \mathrm{e}-2$ & 0.67 & 0.12 & $5.7 e-3$ & 0.54 & 0.43 & $1.8 \mathrm{e}-2$ & 0.65 & 0.29 \\
\hline 50 & 6 & $-3.5 \mathrm{e}-3$ & 0.43 & 0.12 & $4.8 \mathrm{e}-3$ & 0.54 & 0.48 & $1.3 \mathrm{e}-3$ & 0.49 & 0.29 \\
\hline 100 & 6 & $2.0 \mathrm{e}-4$ & 0.48 & 0.12 & $4.6 \mathrm{e}-3$ & 0.52 & 0.48 & $4.7 \mathrm{e}-3$ & 0.52 & 0.31 \\
\hline 500 & 6 & $1.2 \mathrm{e}-2$ & 0.66 & 0.12 & $8.7 e-4$ & 0.45 & 0.49 & $1.3 \mathrm{e}-2$ & 0.62 & 0.34 \\
\hline 50 & 10 & $-2.0 \mathrm{e}-3$ & 0.44 & 0.12 & $3.2 \mathrm{e}-3$ & 0.50 & 0.50 & $1.1 \mathrm{e}-3$ & 0.48 & 0.32 \\
\hline 100 & 10 & $1.8 \mathrm{e}-3$ & 0.49 & 0.12 & $2.4 \mathrm{e}-3$ & 0.48 & 0.50 & $4.2 \mathrm{e}-3$ & 0.52 & 0.34 \\
\hline \multirow[t]{2}{*}{500} & 10 & $1.4 \mathrm{e}-2$ & 0.70 & 0.12 & $2.8 \mathrm{e}-4$ & 0.44 & 0.50 & $1.5 \mathrm{e}-2$ & 0.64 & 0.36 \\
\hline & & \multicolumn{9}{|c|}{$\mathrm{s} 10: \lambda_{0}=0.2, \lambda=5, \Lambda=0.2\left(\sigma_{u_{0}}=0.04 \sigma_{u}=0.2, \sigma_{v_{0}}=0.2, \sigma_{v}=0.04\right)$} \\
\hline 50 & 3 & $-8.6 \mathrm{e}-3$ & 0.52 & 0.10 & $2.8 \mathrm{e}-2$ & 0.56 & 0.81 & $1.9 \mathrm{e}-2$ & 0.54 & 0.77 \\
\hline 100 & 3 & $-4.3 \mathrm{e}-3$ & 0.54 & 0.11 & $1.4 \mathrm{e}-2$ & 0.51 & 0.82 & $1.0 \mathrm{e}-2$ & 0.51 & 0.80 \\
\hline 500 & 3 & $2.4 \mathrm{e}-3$ & 0.59 & 0.11 & $3.2 \mathrm{e}-3$ & 0.46 & 0.83 & $5.5 \mathrm{e}-3$ & 0.49 & 0.81 \\
\hline 50 & 6 & $-1.0 \mathrm{e}-2$ & 0.43 & 0.12 & $-3.5 \mathrm{e}-3$ & 0.43 & 0.89 & $-1.4 \mathrm{e}-2$ & 0.40 & 0.86 \\
\hline 100 & 6 & $-4.7 e-3$ & 0.48 & 0.12 & $-7.1 \mathrm{e}-3$ & 0.41 & 0.89 & $-1.2 \mathrm{e}-2$ & 0.41 & 0.87 \\
\hline 500 & 6 & $6.3 \mathrm{e}-3$ & 0.61 & 0.11 & $-8.0 \mathrm{e}-3$ & 0.40 & 0.89 & $-1.8 \mathrm{e}-3$ & 0.47 & 0.87 \\
\hline 50 & 10 & $-6.6 \mathrm{e}-3$ & 0.42 & 0.12 & $-1.4 \mathrm{e}-2$ & 0.37 & 0.91 & $-2.0 \mathrm{e}-2$ & 0.35 & 0.89 \\
\hline 100 & 10 & $-2.4 \mathrm{e}-4$ & 0.50 & 0.12 & $-1.4 \mathrm{e}-2$ & 0.36 & 0.91 & $-1.5 \mathrm{e}-2$ & 0.39 & 0.89 \\
\hline 500 & 10 & $9.8 \mathrm{e}-3$ & 0.65 & 0.12 & $-1.5 \mathrm{e}-2$ & 0.35 & 0.91 & $-5.4 \mathrm{e}-3$ & 0.45 & 0.89 \\
\hline
\end{tabular}

In the cases where transient technical inefficiency is low relative to idiosyncratic error term, $\lambda=0.2$, GTRE estimator is very bad in predicting both persistent and overall technical efficiency. The relative bias is small and declines very slowly as $t$ and $n$ increase. The upward bias is small meaning that the estimator tends to underestimate more than overestimate. The correlation coefficient is only 0.1 and does not get better as the sample size increases. The transient technical efficiency is underestimated, but the size of underestimation is very small, ranging from $4.1 \%$ for $n=50, t=3$ to $0.91 \%$ for $n=500, t=10$. The upward bias is virtually at the desired level of 0.5. The correlation coefficient is very low and is at par with that for persistent and overall technical efficiency.

$\lambda=1$ : Here the performance of the estimator for all three types of technical efficiency is about the same. In terms of correlation the estimation of transient technical efficiency becomes better. In terms of relative bias, the GTRE estimator performs better in the case of $\Lambda=1$ (s9) than $\Lambda=0.2$ (s14). Although the prediction of transient technical efficiency when $\Lambda=1$ is very good even for small $n$ and $t$, the relative bias of persistent technical efficiency becomes small only when sample size increases. The relative bias of transient technical efficiency is fairly small when $\Lambda=0.2$. If not for correlation of 0.5 , the estimation of transient technical efficiency would be good.

Finally, if $\lambda=5$ (s10), the performance of the estimator for transient technical efficiency gets even better, although GTRE estimator tends to underestimate more when sample size grows. 
Whereas relative bias and upward bias remain virtually the same, the correlation increases from about 0.5 to 0.9 . Similarly to $\mathrm{s} 9$, the relative bias of persistent technical efficiency is quite small. The overall technical efficiency is estimated relatively precisely only when sample size is very large. The correlation between true and estimated overall technical efficiency is up to 0.9 due to high correlation between true and estimated transient technical efficiency.

In sum, Table 3 results suggest that when $\sigma_{u_{0}}$ is low relative to $\sigma_{v_{0}}$, the GTRE estimator performs very poorly in terms of predicting persistent technical efficiency when taking all three criteria into account. The relative bias decreases slowly when sample size increases, whereas the correlation stays about 0.1 no matter what scenario we are looking at. The transient technical efficiency is estimated much better. The relative bias is small even in small sample, the upward bias is approximately 0.5, while correlation increases when there is "more" transient technical inefficiency.

Table 4 presents the comparative measures when $\sigma_{u_{0}}$ equals $\sigma_{v_{0}}$. As before, it is worthwhile to split discussion of the results by $\lambda$ and $\Lambda$.

Table 4: Finite Sample Performance of the Technical Efficiency Estimates. $\sigma_{u_{0}}$ equals $\sigma_{v_{0}}$, i.e., $\lambda_{0}=1$.

\begin{tabular}{|c|c|c|c|c|c|c|c|c|c|c|}
\hline \multirow[b]{2}{*}{$n$} & \multirow[b]{2}{*}{$t$} & \multicolumn{3}{|c|}{ Persistent TE } & \multicolumn{3}{|c|}{ Transient TE } & \multicolumn{3}{|c|}{ Overall TE } \\
\hline & & R Bias & U Bias & Corr & R Bias & U Bias & Corr & R Bias & U Bias & Corr \\
\hline & & \multicolumn{9}{|c|}{$\mathrm{s} 5: \lambda_{0}=1, \lambda=0.2, \Lambda=1\left(\sigma_{u_{0}}=0.04 \sigma_{u}=0.04, \sigma_{v_{0}}=0.04, \sigma_{v}=0.2\right)$} \\
\hline 50 & 3 & $8.3 \mathrm{e}-3$ & 0.68 & 0.19 & $-2.9 \mathrm{e}-2$ & 0.51 & 0.11 & $-2.1 \mathrm{e}-2$ & 0.48 & 0.17 \\
\hline 100 & 3 & $6.4 \mathrm{e}-3$ & 0.64 & 0.20 & $-2.5 \mathrm{e}-2$ & 0.49 & 0.11 & $-1.9 \mathrm{e}-2$ & 0.46 & 0.17 \\
\hline 500 & 3 & $3.1 \mathrm{e}-3$ & 0.56 & 0.19 & $-1.2 \mathrm{e}-2$ & 0.52 & 0.12 & $-8.4 \mathrm{e}-3$ & 0.48 & 0.17 \\
\hline 50 & 6 & $4.7 \mathrm{e}-3$ & 0.61 & 0.25 & $-2.7 \mathrm{e}-2$ & 0.49 & 0.12 & $-2.2 \mathrm{e}-2$ & 0.45 & 0.18 \\
\hline 100 & 6 & $4.6 \mathrm{e}-3$ & 0.59 & 0.25 & $-2.0 \mathrm{e}-2$ & 0.50 & 0.12 & $-1.5 \mathrm{e}-2$ & 0.46 & 0.19 \\
\hline 500 & 6 & $2.3 \mathrm{e}-3$ & 0.53 & 0.26 & $-9.4 \mathrm{e}-3$ & 0.51 & 0.12 & $-7.1 \mathrm{e}-3$ & 0.46 & 0.19 \\
\hline 50 & 10 & $4.5 \mathrm{e}-3$ & 0.58 & 0.30 & $-2.2 \mathrm{e}-2$ & 0.49 & 0.12 & $-1.7 \mathrm{e}-2$ & 0.45 & 0.20 \\
\hline 100 & 10 & $3.0 \mathrm{e}-3$ & 0.55 & 0.31 & $-1.4 \mathrm{e}-2$ & 0.52 & 0.12 & $-1.1 \mathrm{e}-2$ & 0.46 & 0.20 \\
\hline \multirow[t]{2}{*}{500} & 10 & $6.2 \mathrm{e}-3$ & 0.58 & 0.31 & $-6.1 \mathrm{e}-3$ & 0.52 & 0.12 & $6.7 \mathrm{e}-5$ & 0.51 & 0.20 \\
\hline & \multicolumn{10}{|c|}{$\mathrm{s} 15: \lambda_{0}=1, \lambda=0.2, \Lambda=5\left(\sigma_{u_{0}}=0.2 \sigma_{u}=0.04, \sigma_{v_{0}}=0.2, \sigma_{v}=0.2\right)$} \\
\hline 50 & 3 & $3.4 \mathrm{e}-2$ & 0.56 & 0.46 & $-3.9 \mathrm{e}-2$ & 0.49 & 0.10 & $-6.1 \mathrm{e}-3$ & 0.46 & 0.37 \\
\hline 100 & 3 & $3.0 \mathrm{e}-2$ & 0.54 & 0.47 & $-2.9 \mathrm{e}-2$ & 0.50 & 0.10 & $-4.6 \mathrm{e}-4$ & 0.46 & 0.39 \\
\hline 500 & 3 & $3.9 \mathrm{e}-2$ & 0.55 & 0.47 & $-1.9 \mathrm{e}-2$ & 0.50 & 0.10 & $1.9 \mathrm{e}-2$ & 0.50 & 0.40 \\
\hline 50 & 6 & $2.9 \mathrm{e}-2$ & 0.54 & 0.49 & $-3.1 \mathrm{e}-2$ & 0.48 & 0.11 & $-2.9 \mathrm{e}-3$ & 0.46 & 0.39 \\
\hline 100 & 6 & $2.7 \mathrm{e}-2$ & 0.53 & 0.49 & $-2.3 \mathrm{e}-2$ & 0.49 & 0.11 & $3.7 \mathrm{e}-3$ & 0.47 & 0.41 \\
\hline 500 & 6 & $3.3 \mathrm{e}-2$ & 0.53 & 0.49 & $-1.4 \mathrm{e}-2$ & 0.50 & 0.11 & $1.9 \mathrm{e}-2$ & 0.50 & 0.42 \\
\hline 50 & 10 & $2.0 \mathrm{e}-2$ & 0.51 & 0.50 & $-2.5 \mathrm{e}-2$ & 0.49 & 0.11 & $-5.5 \mathrm{e}-3$ & 0.45 & 0.42 \\
\hline 100 & 10 & $2.7 \mathrm{e}-2$ & 0.52 & 0.50 & $-2.0 \mathrm{e}-2$ & 0.49 & 0.11 & $7.4 \mathrm{e}-3$ & 0.47 & 0.43 \\
\hline 500 & 10 & $2.9 \mathrm{e}-2$ & 0.52 & 0.51 & $-9.3 \mathrm{e}-3$ & 0.50 & 0.11 & $1.9 \mathrm{e}-2$ & 0.50 & 0.44 \\
\hline
\end{tabular}


Table 4 (Continued)

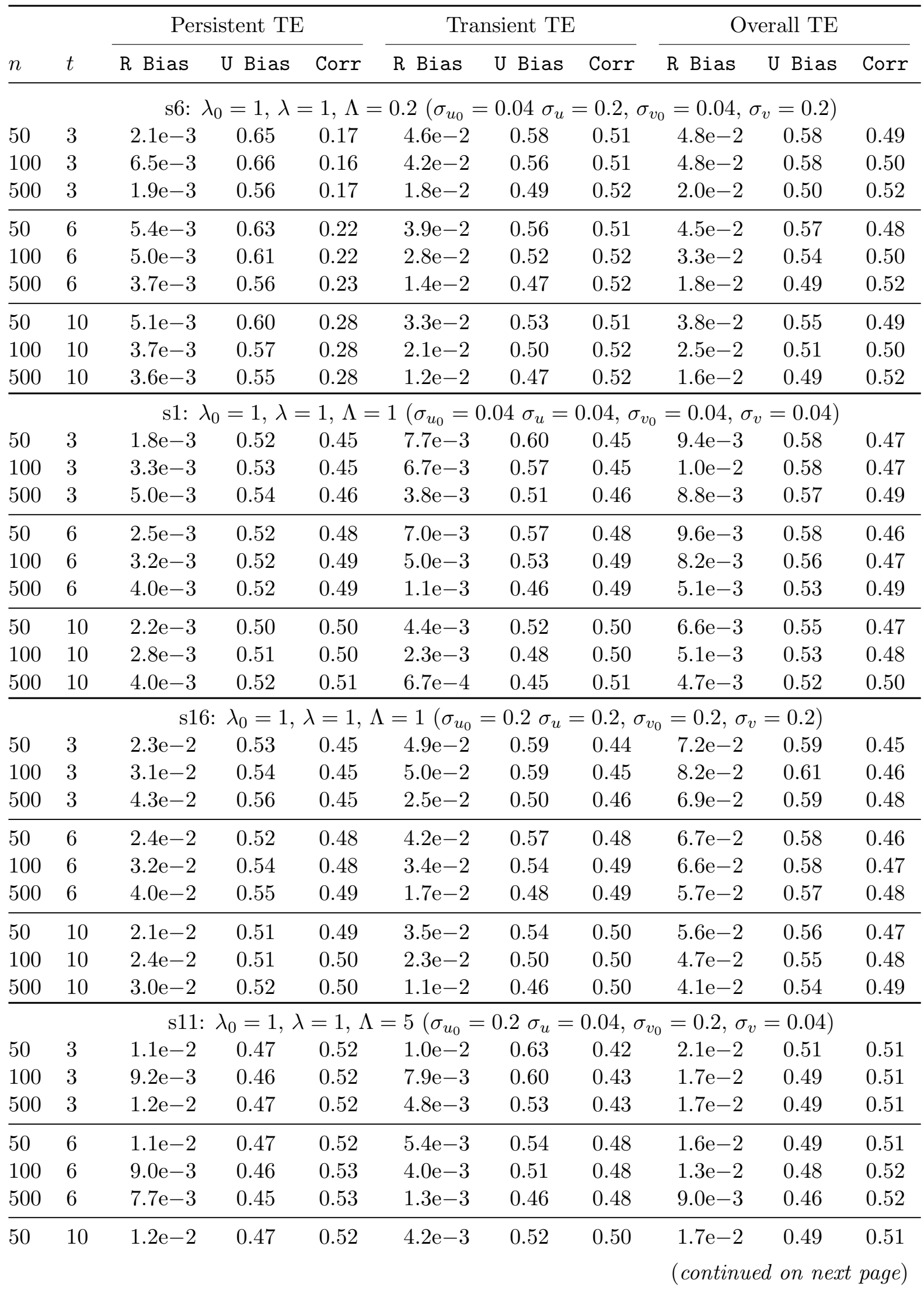


Table 4 (Continued)

\begin{tabular}{|c|c|c|c|c|c|c|c|c|c|c|}
\hline \multirow[b]{2}{*}{$n$} & \multirow[b]{2}{*}{$t$} & \multicolumn{3}{|c|}{ Persistent TE } & \multicolumn{3}{|c|}{ Transient TE } & \multicolumn{3}{|c|}{ Overall TE } \\
\hline & & R Bias & U Bias & Corr & R Bias & U Bias & Corr & R Bias & U Bias & Corr \\
\hline 100 & 10 & $7.2 \mathrm{e}-3$ & 0.45 & 0.52 & $2.9 \mathrm{e}-3$ & 0.49 & 0.50 & $1.0 \mathrm{e}-2$ & 0.46 & 0.52 \\
\hline \multirow[t]{2}{*}{500} & 10 & $5.5 \mathrm{e}-3$ & 0.45 & 0.52 & $5.3 \mathrm{e}-4$ & 0.45 & 0.50 & $6.0 \mathrm{e}-3$ & 0.45 & 0.51 \\
\hline & & \multicolumn{9}{|c|}{$\mathrm{s} 2: \lambda_{0}=1, \lambda=5, \Lambda=0.2\left(\sigma_{u_{0}}=0.04 \sigma_{u}=0.2, \sigma_{v_{0}}=0.04, \sigma_{v}=0.04\right)$} \\
\hline 50 & 3 & $1.6 \mathrm{e}-3$ & 0.55 & 0.29 & $1.0 \mathrm{e}-2$ & 0.53 & 0.90 & $1.1 \mathrm{e}-2$ & 0.55 & 0.90 \\
\hline 100 & 3 & $-1.8 \mathrm{e}-3$ & 0.50 & 0.30 & $5.3 \mathrm{e}-3$ & 0.51 & 0.90 & $3.0 \mathrm{e}-3$ & 0.50 & 0.91 \\
\hline 500 & 3 & $2.1 \mathrm{e}-3$ & 0.51 & 0.30 & $4.0 \mathrm{e}-3$ & 0.50 & 0.91 & $5.7 \mathrm{e}-3$ & 0.52 & 0.91 \\
\hline 50 & 6 & $-3.5 \mathrm{e}-4$ & 0.51 & 0.37 & $2.9 \mathrm{e}-3$ & 0.49 & 0.91 & $2.3 e-3$ & 0.50 & 0.91 \\
\hline 100 & 6 & $1.7 \mathrm{e}-3$ & 0.52 & 0.38 & $1.8 \mathrm{e}-3$ & 0.48 & 0.92 & $3.2 \mathrm{e}-3$ & 0.51 & 0.91 \\
\hline 500 & 6 & $4.9 \mathrm{e}-3$ & 0.54 & 0.38 & $1.5 \mathrm{e}-3$ & 0.48 & 0.92 & $6.1 \mathrm{e}-3$ & 0.53 & 0.92 \\
\hline 50 & 10 & $3.0 \mathrm{e}-3$ & 0.55 & 0.42 & $-1.7 e-3$ & 0.46 & 0.92 & $1.1 \mathrm{e}-3$ & 0.49 & 0.92 \\
\hline 100 & 10 & $1.7 \mathrm{e}-3$ & 0.52 & 0.42 & $-2.1 \mathrm{e}-3$ & 0.45 & 0.93 & $-6.8 \mathrm{e}-4$ & 0.48 & 0.92 \\
\hline \multirow[t]{2}{*}{500} & 10 & $5.7 \mathrm{e}-3$ & 0.55 & 0.42 & $-2.8 \mathrm{e}-3$ & 0.45 & 0.93 & $2.7 \mathrm{e}-3$ & 0.50 & 0.92 \\
\hline & & \multicolumn{9}{|c|}{$\mathrm{s} 12: \lambda_{0}=1, \lambda=5, \Lambda=1\left(\sigma_{u_{0}}=0.2 \sigma_{u}=0.2, \sigma_{v_{0}}=0.2, \sigma_{v}=0.04\right)$} \\
\hline 50 & 3 & $1.8 \mathrm{e}-2$ & 0.50 & 0.50 & $3.6 \mathrm{e}-2$ & 0.59 & 0.81 & $5.3 \mathrm{e}-2$ & 0.59 & 0.67 \\
\hline 100 & 3 & $1.8 \mathrm{e}-2$ & 0.50 & 0.50 & $1.8 \mathrm{e}-2$ & 0.53 & 0.82 & $3.6 \mathrm{e}-2$ & 0.55 & 0.69 \\
\hline 500 & 3 & $2.6 \mathrm{e}-2$ & 0.51 & 0.50 & $4.6 \mathrm{e}-3$ & 0.46 & 0.82 & $3.0 \mathrm{e}-2$ & 0.53 & 0.70 \\
\hline 50 & 6 & $1.9 \mathrm{e}-2$ & 0.50 & 0.51 & $-5.6 \mathrm{e}-3$ & 0.42 & 0.88 & $1.3 \mathrm{e}-2$ & 0.48 & 0.73 \\
\hline 100 & 6 & $1.8 \mathrm{e}-2$ & 0.49 & 0.51 & $-9.1 \mathrm{e}-3$ & 0.40 & 0.88 & $8.7 e-3$ & 0.47 & 0.73 \\
\hline 500 & 6 & $2.3 \mathrm{e}-2$ & 0.50 & 0.51 & $-1.0 \mathrm{e}-2$ & 0.39 & 0.89 & $1.2 \mathrm{e}-2$ & 0.48 & 0.73 \\
\hline 50 & 10 & $1.9 \mathrm{e}-2$ & 0.49 & 0.50 & $-1.6 e-2$ & 0.35 & 0.90 & $1.7 \mathrm{e}-3$ & 0.45 & 0.74 \\
\hline 100 & 10 & $2.0 \mathrm{e}-2$ & 0.50 & 0.51 & $-1.7 e-2$ & 0.34 & 0.91 & $2.6 \mathrm{e}-3$ & 0.45 & 0.74 \\
\hline 500 & 10 & $2.2 \mathrm{e}-2$ & 0.50 & 0.52 & $-1.7 e-2$ & 0.34 & 0.91 & $4.3 \mathrm{e}-3$ & 0.45 & 0.74 \\
\hline
\end{tabular}

If $\lambda=0.2$ and $\Lambda=1$ (s5), the correlation between the true and estimated technical efficiency is very low for all types of technical efficiency, $0.1-0.3$. The upward bias is though about the desired level of 0.5. Transient and overall technical efficiency are generally underestimated, while persistent technical efficiency is overestimated. As expected, the relative bias gets smaller with larger sample size. Estimation of transient technical efficiency is fair for both $\Lambda=1$ (s5) and $\Lambda=5(\mathrm{~s} 15)$.

When $\lambda=1$ and $\Lambda=0.2$ (s6), the relative biases of the estimated transient and overall technical efficiency are small and decreasing in both $n$ and $t$. The upward bias and correlation is about 0.5. The relative bias of persistent technical efficiency is on 'average' even smaller than that of transient technical efficiency. Persistent technical efficiency is underestimated by only $0.2-0.6 \%$. For all three types of technical efficiency, the upward bias achieves the 0.5-mark.

If $\lambda=1$ and $\Lambda=1$ (s1, s16), the upward bias and correlation are nearly identical for all types of technical efficiency for each $n$ and $t$, and both reach approximately 0.5. In terms of relative bias, all types of technical efficiency are estimated a little better in scenario s1. This is 
because in scenario s16 the true technical efficiency vary by much more.

If $\lambda=1$ and $\Lambda=5$ (s11), the performance of GRTE estimator is very similar to that of $\Lambda=1$ (s16) except for an improved relative bias for transient technical efficiency, especially for large $n t$.

When $\lambda=5$ and $\Lambda=0.2$ (s2), transient technical efficiency is estimated to be very good by any measure. The correlation coefficient reaches 0.9 , something we have observed in scenario s10, where $\lambda=5$. Interestingly, transient technical efficiency is overestimated for $t=3,6$ but underestimated for $t=10$ (the level of under- or overestimation is about $0.2 \%$ ). Overall technical efficiency preserves very high correlation coefficient from transient technical efficiency. For all types of technical efficiency, the performance of the GTRE estimator of is marked by a very small relative bias. The relative bias of persistent technical efficiency is in fact slightly increasing in sample size. ${ }^{6}$ The explanation for this phenomena will be become apparent in the next section. The correlation for persistent technical efficiency reaches only 0.4 , while for transient and overall technical efficiency it is over 0.9.

Finally, if $\lambda=5$ and $\Lambda=1$ (s12), persistent and overall technical efficiency are estimated worse than transient technical efficiency in terms of relative bias. Besides, as we observed before, the relative bias tends to increase with sample size. The correlation is about 0.5 and 0.7 respectively. The upward bias is close to 0.5 for large $n t$. The performance of the estimator for transient technical efficiency is much better. The correlation, as in previously discussed scenario $\mathrm{s} 11$, is about 0.9 . Similar to $\mathrm{s} 2$, the transient technical efficiency is overestimated for small $t$ but underestimated for larger $t \mathrm{~s}$.

Table 5 presents the comparative measures when $\sigma_{u_{0}}$ is bigger than $\sigma_{v_{0}}, \lambda_{0}=5$. We observe three cases of $\lambda$. In all three cases, the performance of persistent technical efficiency is good by all chosen measures. The relative bias is small even for small values of $n$ and $t$ and decreases even further as $n$ and $t$ grow. The representative level of misestimation is $1 \%$, but can be as low as $0.034 \%$ for large $n t$ or as high as $4 \%$ for low $n t$. The correlation between true and estimated persistent technical efficiency ranges from 0.7 to 0.9 . As $n$ (irrespective of $t$ ) increases, upward bias achieves the desired level of 0.5 .

Table 5: Finite Sample Performance of the Technical Efficiency Estimates. $\sigma_{u_{0}}>\sigma_{v_{0}}$, i.e., $\lambda_{0}=5$.

\begin{tabular}{|c|c|c|c|c|c|c|c|c|c|c|}
\hline \multirow[b]{2}{*}{$n$} & \multirow[b]{2}{*}{$t$} & \multicolumn{3}{|c|}{ Persistent TE } & \multicolumn{3}{|c|}{ Transient TE } & \multicolumn{3}{|c|}{ Overall TE } \\
\hline & & R Bias & U Bias & Corr & R Bias & U Bias & Corr & R Bias & U Bias & Corr \\
\hline & & s7: & $0=5, \lambda$ & $=0.2, \Lambda$ & $=5\left(\sigma_{u_{0}}\right.$ & $=0.2 \sigma_{u}=$ & 0.04, & $v_{0}=0.04$, & $v=0.2)$ & \\
\hline 50 & 3 & $4.9 \mathrm{e}-2$ & 0.61 & 0.69 & $-3.9 \mathrm{e}-2$ & 0.47 & 0.11 & $7.7 \mathrm{e}-3$ & 0.49 & 0.60 \\
\hline 100 & 3 & $3.5 \mathrm{e}-2$ & 0.56 & 0.71 & $-3.4 \mathrm{e}-2$ & 0.46 & 0.11 & $-5.8 \mathrm{e}-4$ & 0.46 & 0.63 \\
\hline 500 & 3 & $1.3 \mathrm{e}-2$ & 0.49 & 0.71 & $-1.8 \mathrm{e}-2$ & 0.49 & 0.11 & $-5.4 \mathrm{e}-3$ & 0.44 & 0.69 \\
\hline
\end{tabular}

\footnotetext{
${ }^{6}$ Note that relative MSE (not reported here) is decreasing as expected.
} 
Table 5 (Continued)

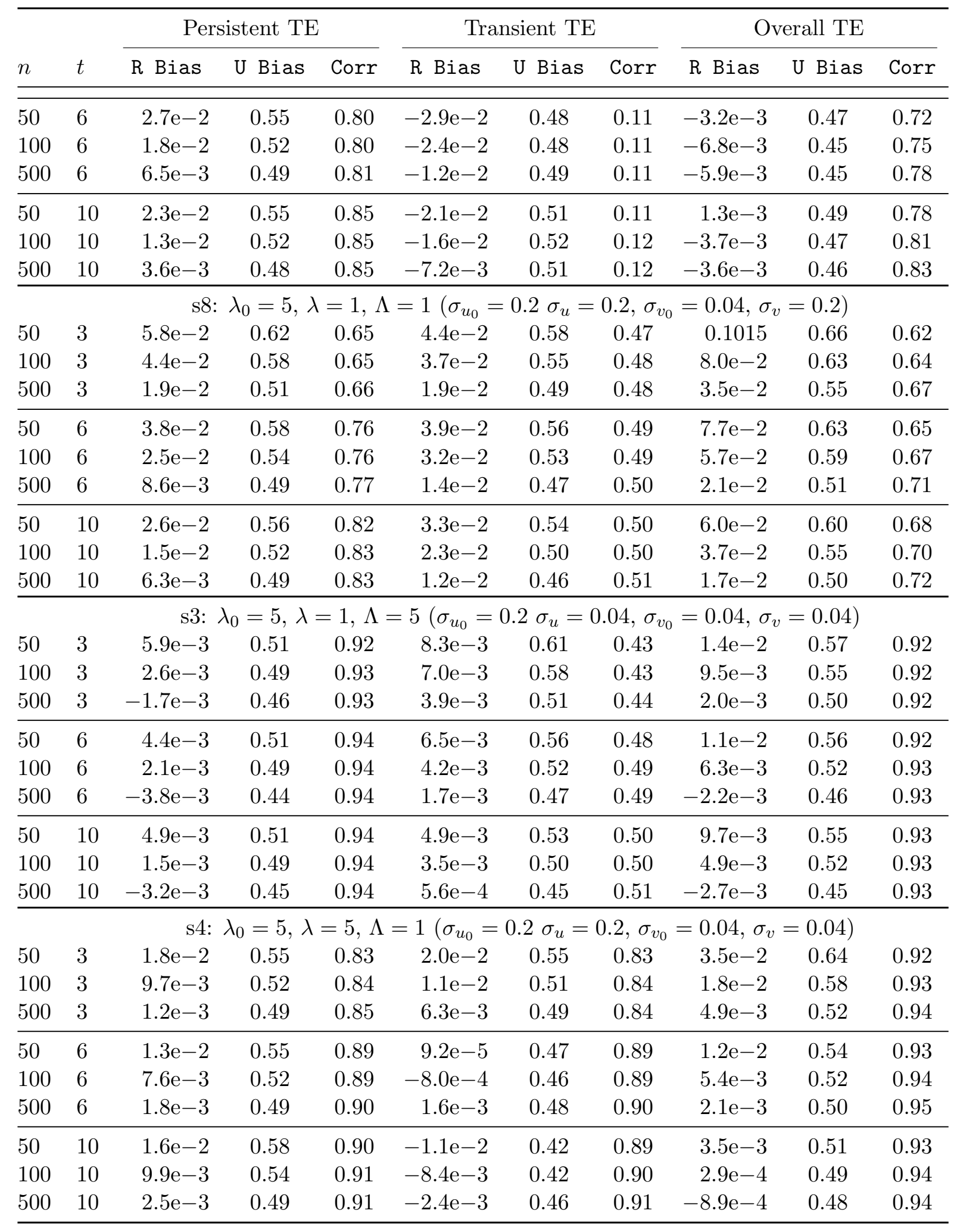

The finite sample properties of transient and overall technical efficiency vary by $\lambda$ and $\Lambda$. 
When $\lambda=0.2(\mathrm{~s} 7)$, the correlation between true and estimated transient technical efficiency is very low, 0.1. The relative biases of both persistent and transient technical efficiency are small, but decrease only very slowly in both $n$ and $t$. Relative bias of overall technical efficiency is in fact slightly increasing. Transient technical efficiency is underestimated on 'average.'

$\lambda=1$. In case of $\Lambda=1$ (s8) the performance of overall technical efficiency estimates is even worse than in s7. Properties of transient technical efficiency are in contrast slightly better: the correlation is 0.5 . Switching to $\Lambda=5$ (s3) makes transient and overall technical efficiency estimates better in terms of relative bias. Transient technical efficiency is overestimated on 'average.' The correlation for overall technical efficiency gets better mostly due to astonishing correlation for persistent technical efficiency of 0.94 .

It is safe to say that by all chosen measured the case of $\lambda=5$ and $\Lambda=1$ (s4) is by far the best in terms of performance of all three types of technical efficiency. The relative bias is small and decreasing in both $n$ and $t$. The upward bias reaches 0.5 for large sample size and correlation is over 0.9 , being 0.95 for overall technical efficiency.

It is early to make generalization at this point, but it is worth noting that if we consider the comparison measures jointly, the persistent technical efficiency and transient technical efficiency can be estimated reliably (consistent, not overly overestimated, and correlated) on 'average' in some scenarios. Table 6 provides a list for such scenarios. Note that good implies that all three measures including correlation between the true and estimated technical efficiency are decent. If correlation coefficient is only 0.5 , the reliability is labeled only "fair."

The transient technical efficiency can be estimated relatively reliably more often than the persistent technical efficiency. Further, for persistent technical efficiency $\lambda_{0}$ should be at least 1 , meaning that the model cannot deal with situation where persistent technical inefficiency is virtually nonexistent relative to random effects. The reliability is only fair for $\lambda_{0}=1$ and it gets good when $\lambda_{0}=5$. Finally, the reliability gets better when persistent technical efficiency prevails over transient technical efficiency ( $\Lambda$ getting bigger). For example, persistent technical efficiency is estimated better in scenario s11 than in scenario s16. By the same token, persistent technical efficiency is estimated better in scenario s3 than in scenario s8.

Almost symmetrically, for reliable transient technical efficiency estimates $\lambda$ should be at least 1 . For example s10, s9, and s6 are absolutely symmetric to s7, s5, and s11, respectively. If $\lambda=1$, transient technical efficiency is estimated to be fair. The estimation of transient technical efficiency becomes good when $\lambda$ increases. As before, the reliability gets better when transient technical efficiency prevails over persistent technical efficiency ( $\Lambda$ getting smaller). The finite sample properties of transient technical efficiency in scenario s1 are better than in s11, in s6 better than in s16, in $\mathrm{s} 2$ - better than in s12.

Table 7 summarizes the results and tells us which scenarios give reliable estimates of technical efficiency. It is clear that in none of the scenarios both persistent technical efficiency and transient technical efficiency can be estimated reliably simultaneously, except for s4, where the 'amount' of noise and random effects is negligible. This is a situation that is next to impossible 
Table 6: Reliability of Efficiency Estimates based on Joint Goodness of all four Comparison Measures

\begin{tabular}{|c|c|c|c|c|c|c|c|c|c|}
\hline \multicolumn{5}{|c|}{ Persistent technical efficiency } & \multicolumn{5}{|c|}{ Transient technical efficiency } \\
\hline Scenario & $\lambda_{0}$ & $\lambda$ & $\Lambda$ & Reliability & Scenario & $\lambda_{0}$ & $\lambda$ & $\Lambda$ & Reliability \\
\hline \multicolumn{10}{|c|}{$\lambda_{0}=0.2$} \\
\hline & & & & & s9: & 0.2 & 1 & 1 & fair \\
\hline & & & & & s10: & 0.2 & 5 & 0.2 & good \\
\hline \multicolumn{10}{|c|}{$\lambda_{0}=1$} \\
\hline s5: & 1 & 0.2 & 1 & fair & & & & & \\
\hline s6: & 1 & 1 & 0.2 & fair & s6: & 1 & 1 & 0.2 & fair \\
\hline s1: & 1 & 1 & 1 & fair & s1: & 1 & 1 & 1 & fair \\
\hline s16: & 1 & 1 & 1 & fair & s16: & 1 & 1 & 1 & fair \\
\hline s11: & 1 & 1 & 5 & fair & s11: & 1 & 1 & 5 & fair \\
\hline s2: & 1 & 5 & 0.2 & fair & s2: & 1 & 5 & 0.2 & good \\
\hline s12: & 1 & 5 & 1 & fair & s12: & 1 & 5 & 1 & good \\
\hline \multicolumn{10}{|c|}{$\lambda_{0}=5$} \\
\hline s7: & 5 & 0.2 & 5 & good & & & & & \\
\hline s8: & 5 & 1 & 1 & good & s8: & 5 & 1 & 1 & fair \\
\hline s3: & 5 & 1 & 5 & good & s3: & 5 & 1 & 5 & fair \\
\hline s4: & 5 & 5 & 1 & good & s4: & 5 & 5 & 1 & good \\
\hline
\end{tabular}

to get in real life. Other than scenario s4, one can hope to estimate either persistent technical efficiency or transient technical efficiency relatively reliably.

Table 7 also suggests that judging the reliability of the estimator cannot be based only on two parameters $\lambda_{0}$ and $\lambda$. Parameters lambda ( $\lambda_{0}$ for persistent technical efficiency and $\lambda$ for transient technical efficiency) help in determining the finite sample properties of the estimator. Finally, the practitioner should place relatively little confidence in efficiency estimates from either technical efficiency estimator when respective lambda values are relatively small.

\subsubsection{Percentiles of technical efficiency}

It is difficult to conclude from the tables whether or not the estimator underestimates more or less, and how it performs away from the middle of the distribution. Therefore, we look at other percentiles of the efficiency distribution as well. Specifically, we report the mean of the relative deviation between the 5th, 50th and 95th percentiles of the estimated and true technical efficiency. For tractability, we break down the results by $\lambda_{0}$ and report mean relative deviation for the 5th, median, and 95th percentiles of the technical efficiency estimates in Tables 8-10. We discuss only scenarios where technical efficiency is estimated to be at least fair according to Table 6. 
Table 7: Scenarios with Reliable Efficiency Estimates on 'Average'

\begin{tabular}{llll}
\hline & $\lambda=0.2$ & $\lambda=1$ & $\lambda=5$ \\
\cline { 2 - 4 }$\lambda_{0}=0.2$ & $\mathrm{~s} 13$ & {$[\mathrm{~s} 9], \mathrm{s} 14$} & {$[[\mathrm{~s} 10]]$} \\
$\lambda_{0}=1$ & $(\mathrm{~s} 5), \mathrm{s} 15$ & {$[(\mathrm{~s} 6)],[(\mathrm{s} 11)],[(\mathrm{s} 1, \mathrm{~s} 16)]$} & {$[[(\mathrm{s} 2)]],[[(\mathrm{s} 12)]]$} \\
$\lambda_{0}=5$ & $((\mathrm{~s} 7))$ & $((\mathrm{s} 3)),((\mathrm{s} 8))$ & $[((\mathrm{s} 4))]]$ \\
\hline
\end{tabular}

Parentheses denote reliable persistent technical efficiency estimates; brackets denote reliable transient technical efficiency estimates. No parentheses or brackets imply scenario without reliable estimates of either technical efficiency.

Table 8: The Mean of the Relative Deviation Between the 5th, 50th and 95th Percentiles of the Estimated and True Technical Efficiency. $\sigma_{u_{0}}<\sigma_{v_{0}}$, i.e., $\lambda_{0}=0.2$.

\begin{tabular}{|c|c|c|c|c|c|c|c|c|c|c|}
\hline \multirow[b]{2}{*}{$n$} & \multirow[b]{2}{*}{$t$} & \multicolumn{3}{|c|}{ Persistent TE } & \multicolumn{3}{|c|}{ Transient TE } & \multicolumn{3}{|c|}{ Overall TE } \\
\hline & & 5 th & 50 th & 95 th & 5 th & 50 th & 95 th & 5 th & 50 th & 95 th \\
\hline \multicolumn{11}{|c|}{$\mathrm{s} 13: \lambda_{0}=0.2, \lambda=0.2, \Lambda=1\left(\sigma_{u_{0}}=0.04 \sigma_{u}=0.04, \sigma_{v_{0}}=0.2, \sigma_{v}=0.2\right)$} \\
\hline 50 & 3 & $-1.2 \mathrm{e}-2$ & $-2.3 \mathrm{e}-2$ & $-2.7 \mathrm{e}-2$ & $-5.3 \mathrm{e}-2$ & $-4.0 \mathrm{e}-2$ & $-3.5 \mathrm{e}-2$ & $-8.7 \mathrm{e}-2$ & $-5.7 \mathrm{e}-2$ & $-5.3 \mathrm{e}-2$ \\
\hline 100 & 3 & $-2.2 \mathrm{e}-3$ & $-1.9 \mathrm{e}-2$ & $-2.6 \mathrm{e}-2$ & $-3.2 \mathrm{e}-2$ & $-3.3 \mathrm{e}-2$ & $-3.4 \mathrm{e}-2$ & $-5.8 \mathrm{e}-2$ & $-4.7 e-2$ & $-5.1 \mathrm{e}-2$ \\
\hline 500 & 3 & $1.8 \mathrm{e}-2$ & $-1.3 \mathrm{e}-2$ & $-2.5 \mathrm{e}-2$ & $-2.4 \mathrm{e}-3$ & $-2.3 \mathrm{e}-2$ & $-3.1 \mathrm{e}-2$ & $-1.3 \mathrm{e}-2$ & $-3.1 \mathrm{e}-2$ & $-4.6 \mathrm{e}-2$ \\
\hline 50 & 6 & $6.6 \mathrm{e}-4$ & $-1.6 \mathrm{e}-2$ & $-2.4 \mathrm{e}-2$ & $-2.5 \mathrm{e}-2$ & $-2.9 \mathrm{e}$ & $-3.1 \mathrm{e}$ & $-4.7 \epsilon$ & $-4.1 \mathrm{e}-2$ & $-4.7 \mathrm{e}-2$ \\
\hline 100 & 6 & $9.4 \mathrm{e}-3$ & $-1.4 \mathrm{e}-2$ & $-2.4 \mathrm{e}-2$ & $-1.0 \mathrm{e}-2$ & $-2.4 \mathrm{e}-2$ & $-3.0 \mathrm{e}-2$ & $-2.5 \mathrm{e}-2$ & $-3.4 \mathrm{e}-2$ & $-4.5 \mathrm{e}-2$ \\
\hline 500 & 6 & $3.5 \mathrm{e}-2$ & $-2.9 \mathrm{e}-3$ & $-1.9 \mathrm{e}-2$ & $1.5 \mathrm{e}-2$ & $-1.5 \mathrm{e}-2$ & $-2.7 \mathrm{e}-2$ & $2.1 \mathrm{e}-2$ & $-1.3 \mathrm{e}-2$ & $-3.6 \mathrm{e}-2$ \\
\hline 50 & 10 & $1.0 \mathrm{e}-2$ & $-1.4 \mathrm{e}-2$ & $-2.4 \mathrm{e}-2$ & $-1.1 \mathrm{e}-2$ & $-2.5 \mathrm{e}-2$ & $-3.0 \mathrm{e}-2$ & $-2.5 \mathrm{e}-2$ & $-3.4 \mathrm{e}-2$ & $-4.6 \mathrm{e}-2$ \\
\hline 100 & 10 & $1.8 \mathrm{e}-2$ & $-1.1 \mathrm{e}-2$ & $-2.3 \mathrm{e}-2$ & $-2.2 \mathrm{e}-3$ & $-2.2 \mathrm{e}-2$ & $-3.0 \mathrm{e}-2$ & $-1.0 \mathrm{e}-2$ & $-2.8 \mathrm{e}-2$ & $-4.5 \mathrm{e}-2$ \\
\hline 500 & 10 & $4.1 \mathrm{e}-2$ & $-6.1 \mathrm{e}-4$ & $-1.8 \mathrm{e}-2$ & $2.1 \mathrm{e}-2$ & $-1.3 \mathrm{e}-2$ & $-2.6 \mathrm{e}-2$ & $3.3 \mathrm{e}-2$ & $-8.3 \mathrm{e}-3$ & $-3.5 \mathrm{e}-2$ \\
\hline \multicolumn{11}{|c|}{$\mathrm{s} 14: \lambda_{0}=0.2, \lambda=1, \Lambda=0.2\left(\sigma_{u_{0}}=0.04 \sigma_{u}=0.2, \sigma_{v_{0}}=0.2, \sigma_{v}=0.2\right)$} \\
\hline 50 & 3 & $-2.3 \mathrm{e}-2$ & $-2.7 e-2$ & $-2.8 \mathrm{e}-2$ & $1.9 \mathrm{e}-1$ & $3.0 \mathrm{e}-2$ & $-4.3 \mathrm{e}-2$ & $1.2 \mathrm{e}-1$ & $6.0 \mathrm{e}-3$ & $-5.3 \mathrm{e}-2$ \\
\hline 100 & 3 & $-1.0 \mathrm{e}-2$ & $-2.3 \mathrm{e}-2$ & $-2.8 \mathrm{e}-2$ & $2.0 \mathrm{e}-1$ & $2.8 \mathrm{e}-2$ & $-4.6 \mathrm{e}-2$ & $1.5 \mathrm{e}-1$ & $8.8 \mathrm{e}-3$ & $-5.7 \mathrm{e}-2$ \\
\hline 500 & 3 & $1.3 \mathrm{e}-2$ & $-1.5 \mathrm{e}-2$ & $-2.6 \mathrm{e}-2$ & $1.7 \mathrm{e}-1$ & $8.1 \mathrm{e}-3$ & $-6.2 \mathrm{e}-2$ & $1.5 \mathrm{e}-1$ & $-2.6 \mathrm{e}-3$ & $-7.0 \mathrm{e}-2$ \\
\hline 50 & 6 & $-3.2 \mathrm{e}-3$ & $-1.9 \mathrm{e}-2$ & $-2.6 \mathrm{e}-2$ & $1.9 \mathrm{e}-1$ & $2.6 \mathrm{e}-2$ & $-4.8 \mathrm{e}-2$ & $1.5 \mathrm{e}-1$ & $1.1 \mathrm{e}-2$ & $-5.7 \mathrm{e}-2$ \\
\hline 100 & 6 & $8.8 \mathrm{e}-3$ & $-1.5 \mathrm{e}-2$ & $-2.5 \mathrm{e}-2$ & $1.7 \mathrm{e}-1$ & $1.3 \mathrm{e}-2$ & $-5.7 \mathrm{e}-2$ & $1.4 \mathrm{e}-1$ & $2.6 \mathrm{e}-3$ & $-6.5 \mathrm{e}-2$ \\
\hline 500 & 6 & $2.5 \mathrm{e}-2$ & $-8.5 e-3$ & $-2.2 \mathrm{e}-2$ & $1.4 \mathrm{e}-1$ & $-4.0 \mathrm{e}-3$ & $-6.8 \mathrm{e}-2$ & $1.3 \mathrm{e}-1$ & $-8.0 \mathrm{e}-3$ & $-7.4 \mathrm{e}-2$ \\
\hline 50 & 10 & $3.3 e-3$ & $-1.7 \mathrm{e}-2$ & $-2.5 \mathrm{e}-2$ & $1.7 \mathrm{e}-1$ & $1.4 \mathrm{e}-2$ & $-5.6 \mathrm{e}-2$ & $1.4 \mathrm{e}-1$ & $5.4 \mathrm{e}-4$ & $-6.6 \mathrm{e}-2$ \\
\hline 100 & 10 & $1.1 \mathrm{e}-2$ & $-1.5 \mathrm{e}-2$ & $-2.5 \mathrm{e}-2$ & $1.5 \mathrm{e}-1$ & $2.1 \mathrm{e}-3$ & $-6.3 \mathrm{e}-2$ & $1.3 \mathrm{e}-1$ & $-8.5 e-3$ & $-7.3 \mathrm{e}-2$ \\
\hline 500 & 10 & $3.4 \mathrm{e}-2$ & $-4.2 \mathrm{e}-3$ & $-2.0 \mathrm{e}-2$ & $1.3 \mathrm{e}-1$ & $-7.5 \mathrm{e}-3$ & $-6.9 \mathrm{e}-2$ & $1.3 \mathrm{e}-1$ & $-7.0 \mathrm{e}-3$ & $-7.3 \mathrm{e}-2$ \\
\hline \multicolumn{11}{|c|}{$\mathrm{s} 9: \lambda_{0}=0.2, \lambda=1, \Lambda=1\left(\sigma_{u_{0}}=0.04 \sigma_{u}=0.04, \sigma_{v_{0}}=0.2, \sigma_{v}=0.04\right)$} \\
\hline 50 & 3 & $2.7 \mathrm{e}-2$ & $-1.0 \mathrm{e}-2$ & $-2.6 \mathrm{e}-2$ & $3.5 \mathrm{e}-2$ & $6.4 \mathrm{e}-3$ & $-9.0 \mathrm{e}-3$ & $3.7 e-2$ & $1.2 \mathrm{e}-3$ & $-2.6 \mathrm{e}-2$ \\
\hline 100 & 3 & $3.5 \mathrm{e}-2$ & $-6.2 \mathrm{e}-3$ & $-2.3 \mathrm{e}-2$ & $3.3 e-2$ & $4.7 \mathrm{e}-3$ & $-1.0 \mathrm{e}-2$ & $4.2 \mathrm{e}-2$ & $3.5 \mathrm{e}-3$ & $-2.5 \mathrm{e}-2$ \\
\hline 500 & 3 & $5.5 \mathrm{e}-2$ & $7.1 \mathrm{e}-3$ & $-1.4 \mathrm{e}-2$ & $3.4 \mathrm{e}-2$ & $2.5 \mathrm{e}-3$ & $-1.3 \mathrm{e}-2$ & $6.1 \mathrm{e}-2$ & $1.5 \mathrm{e}-2$ & $-1.7 \mathrm{e}-2$ \\
\hline 50 & 6 & $3.2 \mathrm{e}-2$ & $-8.0 \mathrm{e}-3$ & $-2.5 \mathrm{e}-2$ & $2.9 \mathrm{e}-2$ & $2.4 \mathrm{e}-3$ & $-1.1 \mathrm{e}-2$ & $3.7 \mathrm{e}-2$ & $-6.7 e-4$ & $-2.8 \mathrm{e}-2$ \\
\hline 100 & 6 & $3.8 \mathrm{e}-2$ & $-4.5 e-3$ & $-2.3 \mathrm{e}-2$ & $3.0 \mathrm{e}-2$ & $1.8 \mathrm{e}-3$ & $-1.2 \mathrm{e}-2$ & $4.3 \mathrm{e}-2$ & $2.3 \mathrm{e}-3$ & $-2.7 \mathrm{e}-2$ \\
\hline 500 & 6 & $5.6 \mathrm{e}-2$ & $7.1 \mathrm{e}-3$ & $-1.4 \mathrm{e}-2$ & $2.5 \mathrm{e}-2$ & $-1.9 \mathrm{e}-3$ & $-1.5 \mathrm{e}-2$ & $5.3 \mathrm{e}-2$ & $1.1 \mathrm{e}-2$ & $-2.0 \mathrm{e}-2$ \\
\hline
\end{tabular}

(continued on next page) 
Table 8 (Continued)

\begin{tabular}{|c|c|c|c|c|c|c|c|c|c|c|}
\hline \multirow[b]{2}{*}{$n$} & \multirow[b]{2}{*}{$t$} & \multicolumn{3}{|c|}{ Persistent TE } & \multicolumn{3}{|c|}{ Transient TE } & \multicolumn{3}{|c|}{ Overall TE } \\
\hline & & 5 th & 50 th & 95 th & 5 th & 50 th & 95 th & 5 th & 50 th & 95 th \\
\hline 50 & 10 & $3.5 \mathrm{e}-2$ & $-6.7 \mathrm{e}-3$ & $-2.4 \mathrm{e}-2$ & $2.7 \mathrm{e}-2$ & $6.7 \mathrm{e}-4$ & $-1.3 \mathrm{e}-2$ & $3.7 \mathrm{e}-2$ & $-9.9 \mathrm{e}-4$ & $-2.9 \mathrm{e}-2$ \\
\hline 100 & 10 & $4.1 \mathrm{e}-2$ & $-3.1 \mathrm{e}-3$ & $-2.2 \mathrm{e}-2$ & $2.7 \mathrm{e}-2$ & $-2.2 \mathrm{e}-4$ & $-1.3 \mathrm{e}-2$ & $4.3 \mathrm{e}-2$ & $1.8 \mathrm{e}-3$ & $-2.8 \mathrm{e}-2$ \\
\hline 500 & 10 & $5.8 \mathrm{e}-2$ & $9.1 \mathrm{e}-3$ & $-1.3 \mathrm{e}-2$ & $2.4 \mathrm{e}-2$ & $-2.3 \mathrm{e}-3$ & $-1.5 \mathrm{e}-2$ & $5.4 \mathrm{e}-2$ & $1.2 \mathrm{e}-2$ & $-1.8 \mathrm{e}-2$ \\
\hline \multicolumn{11}{|c|}{ s10: $\lambda_{0}=0.2, \lambda=5, \Lambda=0.2\left(\sigma_{u_{0}}=0.04 \sigma_{u}=0.2, \sigma_{v_{0}}=0.2, \sigma_{v}=0.04\right)$} \\
\hline 50 & 3 & $1.5 \mathrm{e}-2$ & $-1.2 \mathrm{e}-2$ & $-2.3 \mathrm{e}-2$ & $9.7 \mathrm{e}-2$ & $2.6 \mathrm{e}-2$ & $-3.8 \mathrm{e}-2$ & $8.1 \mathrm{e}-2$ & $1.7 \mathrm{e}-2$ & $-4.4 \mathrm{e}-2$ \\
\hline 100 & 3 & $2.5 \mathrm{e}-2$ & $-8.1 \mathrm{e}-3$ & $-2.2 \mathrm{e}-2$ & $6.1 \mathrm{e}-2$ & $1.7 \mathrm{e}-2$ & $-4.1 \mathrm{e}-2$ & $5.5 \mathrm{e}-2$ & $1.2 \mathrm{e}-2$ & $-4.6 \mathrm{e}-2$ \\
\hline 500 & 3 & $3.9 \mathrm{e}-2$ & $-2.1 \mathrm{e}-3$ & $-1.9 \mathrm{e}-2$ & $2.9 \mathrm{e}-2$ & $9.5 \mathrm{e}-3$ & $-4.2 \mathrm{e}-2$ & $3.3 \mathrm{e}-2$ & $1.2 \mathrm{e}-2$ & $-4.3 \mathrm{e}-2$ \\
\hline 50 & 6 & $1.7 \mathrm{e}-2$ & $-1.4 \mathrm{e}-2$ & $-2.7 \mathrm{e}-2$ & $1.6 \mathrm{e}-2$ & $1.8 \mathrm{e}-5$ & $-3.6 \mathrm{e}-2$ & $5.5 \mathrm{e}-3$ & $-1.0 \mathrm{e}-2$ & $-4.8 \mathrm{e}-2$ \\
\hline 100 & 6 & $2.8 \mathrm{e}-2$ & $-9.0 \mathrm{e}-3$ & $-2.4 \mathrm{e}-2$ & $8.3 \mathrm{e}-3$ & $-3.4 \mathrm{e}-3$ & $-3.6 \mathrm{e}-2$ & $4.4 \mathrm{e}-3$ & $-8.3 e-3$ & $-4.4 \mathrm{e}-2$ \\
\hline 500 & 6 & $4.6 \mathrm{e}-2$ & $1.5 \mathrm{e}-3$ & $-1.7 \mathrm{e}-2$ & $7.3 \mathrm{e}-3$ & $-3.6 \mathrm{e}-3$ & $-3.7 \mathrm{e}-2$ & $1.6 \mathrm{e}-2$ & $3.0 \mathrm{e}-3$ & $-3.7 \mathrm{e}-2$ \\
\hline 50 & 10 & $2.5 \mathrm{e}-2$ & $-1.1 \mathrm{e}-2$ & $-2.6 \mathrm{e}-2$ & $-4.9 \mathrm{e}-3$ & $-1.0 \mathrm{e}-2$ & $-3.5 \mathrm{e}-2$ & $-9.8 \mathrm{e}-3$ & $-1.7 \mathrm{e}-2$ & $-4.7 \mathrm{e}-2$ \\
\hline 100 & 10 & $3.6 \mathrm{e}-2$ & $-4.8 \mathrm{e}-3$ & $-2.2 \mathrm{e}-2$ & $-5.2 \mathrm{e}-3$ & $-1.0 \mathrm{e}-2$ & $-3.7 \mathrm{e}-2$ & $-3.5 \mathrm{e}-3$ & $-1.0 \mathrm{e}-2$ & $-4.3 \mathrm{e}-2$ \\
\hline 500 & 10 & $5.2 \mathrm{e}-2$ & $4.9 \mathrm{e}-3$ & $-1.5 \mathrm{e}-2$ & $-5.3 \mathrm{e}-3$ & $-1.1 \mathrm{e}-2$ & $-3.8 \mathrm{e}-2$ & $6.9 \mathrm{e}-3$ & $-9.1 \mathrm{e}-4$ & $-3.6 \mathrm{e}-2$ \\
\hline
\end{tabular}

Table 8 suggests that for scenario s9 $\left(\lambda_{0}=0.2, \lambda=1, \Lambda=1\right)$, GTRE can predict the median of the transient technical efficiency to be very good, overestimating it by less than $1 \%$. While 95th percentile of transient technical efficiency is underestimated by slightly more than $1 \%$, the 5 th percentile is overestimated by more than $3 \%$. Thus, transient technical efficiency can be estimated quite precisely in the middle, but the estimation is only fair for least and most efficient observations.

We noted previously that 'good' estimation of the 'average' transient technical efficiency in scenario s10, where $\lambda=5$. This is confirmed in Table 8 . For very large values of $n t$ the smallest transient technical efficiency are also predicted to be good, but the largest transient technical efficiency are predicted to be only fair.

Table 9: The Mean of the Relative Deviation Between the 5th, 50th and 95th Percentiles of the Estimated and True Technical Efficiency. $\sigma_{u_{0}}=\sigma_{v_{0}}$, i.e., $\lambda_{0}=1$.

\begin{tabular}{|c|c|c|c|c|c|c|c|c|c|c|}
\hline \multirow[b]{2}{*}{$n$} & \multirow[b]{2}{*}{$t$} & \multicolumn{3}{|c|}{ Persistent TE } & \multicolumn{3}{|c|}{ Transient TE } & \multicolumn{3}{|c|}{ Overall TE } \\
\hline & & 5 th & 50 th & 95 th & 5 th & 50 th & 95 th & 5 th & 50 th & 95 th \\
\hline \multicolumn{11}{|c|}{$\mathrm{s} 5: \lambda_{0}=1, \lambda=0.2, \Lambda=1\left(\sigma_{u_{0}}=0.04 \sigma_{u}=0.04, \sigma_{v_{0}}=0.04, \sigma_{v}=0.2\right)$} \\
\hline 50 & 3 & $3.8 \mathrm{e}-2$ & $4.8 \mathrm{e}-3$ & $-1.1 \mathrm{e}-2$ & $-3.2 \mathrm{e}-2$ & $-2.9 \mathrm{e}-2$ & $-2.9 \mathrm{e}-2$ & $-2.1 \mathrm{e}-2$ & $-2.0 \mathrm{e}-2$ & $-3.0 \mathrm{e}-2$ \\
\hline 100 & 3 & $3.8 \mathrm{e}-2$ & $2.6 \mathrm{e}-3$ & $-1.3 \mathrm{e}-2$ & $-1.9 \mathrm{e}-2$ & $-2.6 \mathrm{e}-2$ & $-2.9 \mathrm{e}-2$ & $-8.7 \mathrm{e}-3$ & $-1.9 \mathrm{e}-2$ & $-3.3 \mathrm{e}-2$ \\
\hline 500 & 3 & $3.6 \mathrm{e}-2$ & $-9.6 \mathrm{e}-4$ & $-1.7 \mathrm{e}-2$ & $1.3 \mathrm{e}-2$ & $-1.5 \mathrm{e}-2$ & $-2.6 \mathrm{e}-2$ & $2.1 \mathrm{e}-2$ & $-1.1 \mathrm{e}-2$ & $-3.3 \mathrm{e}-2$ \\
\hline 50 & 6 & $30 \mathrm{p}$ & 2 & $-1.2 \mathrm{e}$ & -2.3 & -2.8 & -3 . & -1.8 & -2.1 & $-3.3 \mathrm{e}-2$ \\
\hline 100 & 6 & $3.3 \mathrm{e}-2$ & $1.3 \mathrm{e}$ & $-1.3 \mathrm{e}-2$ & $-5.3 \mathrm{e}-3$ & -2 & -2.8 & & $-1.6 \mathrm{e}-2$ & $-3.3 e-2$ \\
\hline 500 & 6 & $3.1 \mathrm{e}-2$ & $-1.2 \mathrm{e}-3$ & $-1.6 \mathrm{e}-2$ & $1.8 \mathrm{e}-2$ & $-1.3 \mathrm{e}-2$ & $-2.5 €$ & 2.2 & $-9.3 \mathrm{e}-3$ & $-3.2 \mathrm{e}-2$ \\
\hline - & 10 & $2.8 \mathrm{e}-2$ & & 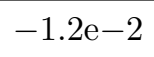 & 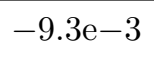 & & & & & 0.8 \\
\hline 100 & 10 & $2.7 \mathrm{e}-2$ & & $1.3 \mathrm{e}$ & 6.7 & re & $-2.6 \mathrm{e}$ & & -1.2 & $-3.1 \mathrm{e}-2$ \\
\hline 500 & 10 & $3.6 \mathrm{e}-2$ & $2.7 \mathrm{e}-3$ & $-1.3 \mathrm{e}-2$ & $2.5 \mathrm{e}-2$ & $-1.0 \mathrm{e}-2$ & $-2.4 \mathrm{e}-2$ & $3.4 \mathrm{e}-2$ & $-2.4 \mathrm{e}-3$ & $-2.7 \mathrm{e}-2$ \\
\hline
\end{tabular}

(continued on next page) 
Table 9 (Continued)

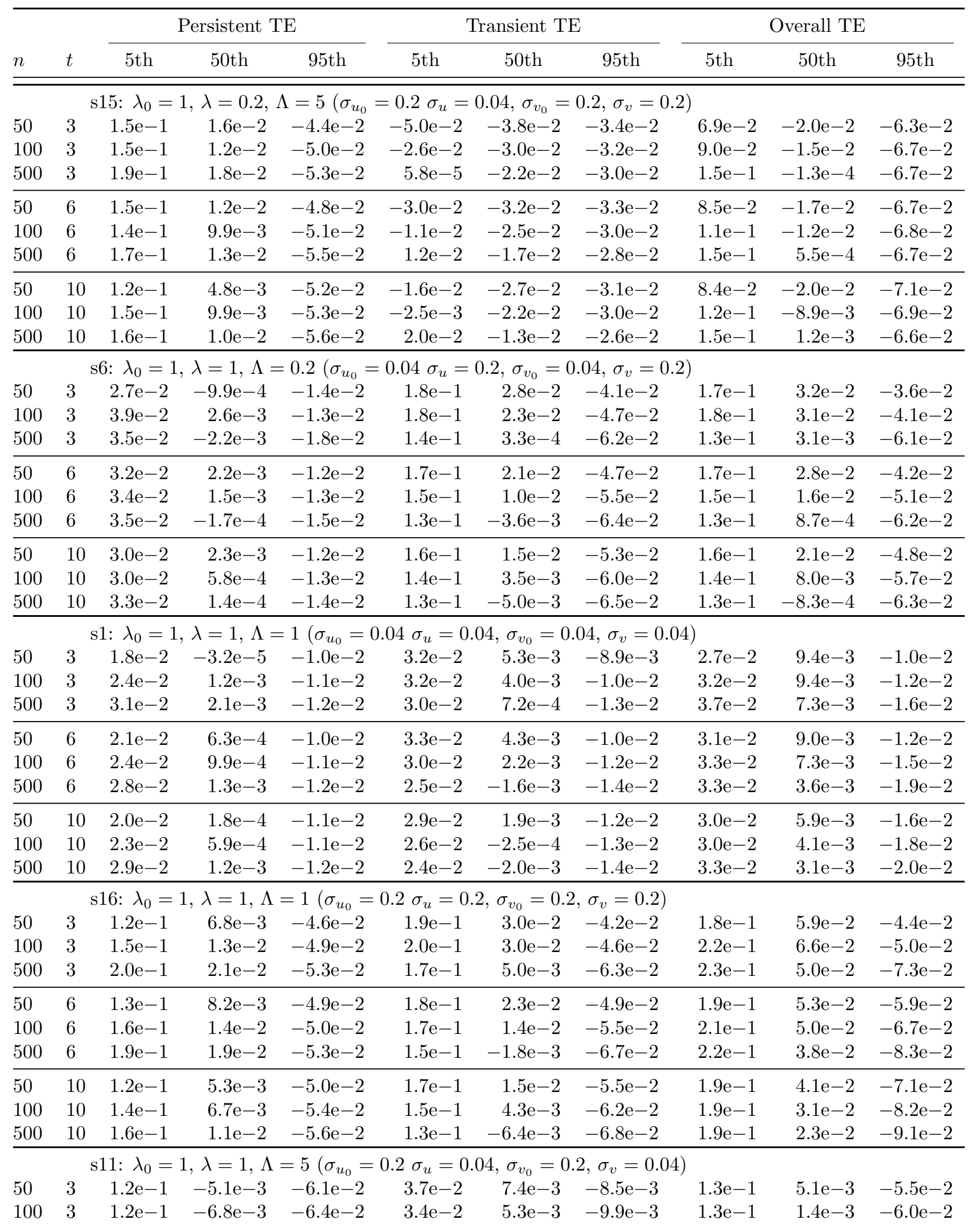

(continued on next page) 
Table 9 (Continued)

\begin{tabular}{|c|c|c|c|c|c|c|c|c|c|c|}
\hline \multirow[b]{2}{*}{$n$} & \multirow[b]{2}{*}{$t$} & \multicolumn{3}{|c|}{ Persistent TE } & \multicolumn{3}{|c|}{ Transient TE } & \multicolumn{3}{|c|}{ Overall TE } \\
\hline & & 5 th & 50 th & 95th & 5 th & 50 th & 95 th & 5 th & 50 th & 95th \\
\hline 500 & 3 & $1.3 \mathrm{e}-1$ & $-6.1 \mathrm{e}-3$ & $6.6 \mathrm{e}-2$ & $3.3 \mathrm{e}-2$ & $1.8 \mathrm{e}-3$ & $-1.3 \mathrm{e}-2$ & $1.4 \mathrm{e}-1$ & $-8.8 \mathrm{e}-4$ & $-6.5 \mathrm{e}-2$ \\
\hline 50 & 6 & $1.2 \mathrm{e}-1$ & $-5.0 \mathrm{e}-3$ & $-6.2 \mathrm{e}-2$ & $3.0 \mathrm{e}-2$ & $2.8 \mathrm{e}-3$ & $-1.1 \mathrm{e}$ & $1.3 \mathrm{e}-1$ & $6.0 \mathrm{e}-4$ & $-6.1 \mathrm{e}-2$ \\
\hline 100 & 6 & $1.2 \mathrm{e}-1$ & $-7.6 \mathrm{e}-3$ & $-6.5 \mathrm{e}$ & $2.9 \mathrm{e}-2$ & $1.3 \mathrm{e}$ & -1.2 & & $-3.3 e-3$ & -6.5 \\
\hline 500 & 6 & $1.3 \mathrm{e}-1$ & $-1.1 \mathrm{e}-2$ & $-7.0 \mathrm{e}-2$ & $2.6 \mathrm{e}-2$ & $-1.5 \mathrm{e}-3$ & $-1.5 \mathrm{e}-2$ & $1.3 \mathrm{e}-1$ & $-8.9 \mathrm{e}-3$ & $-7.2 \mathrm{e}-2$ \\
\hline 50 & 10 & $1.2 \mathrm{e}-1$ & $4.4 \mathrm{e}-3$ & $-6.4 \mathrm{e}-2$ & $2.8 \mathrm{e}-2$ & $1.6 \mathrm{e}-3$ & $-1.2 \mathrm{e}-2$ & $1.3 \mathrm{e}-1$ & $-3.0 \mathrm{e}-4$ & $-6.4 \mathrm{e}-2$ \\
\hline 100 & 10 & $1.2 \mathrm{e}-1$ & -3 & $-6.7 \mathrm{e}$ & $2.7 \mathrm{e}$ & $1.7 \mathrm{e}$ & -1.3 & & -6.8 & -6.8 \\
\hline \multirow[t]{2}{*}{$\underline{500}$} & 10 & $1.3 \mathrm{e}-1$ & $-1.3 \mathrm{e}-2$ & $-7.2 \mathrm{e}-2$ & $2.4 \mathrm{e}-2$ & $-2.2 \mathrm{e}-3$ & $-1.5 \mathrm{e}-2$ & & -1.2 & -7.5 \\
\hline & \multicolumn{9}{|c|}{$\mathrm{s} 2: \lambda_{0}=1, \lambda=5, \Lambda=0.2\left(\sigma_{u_{0}}=0.04 \sigma_{u}=0.2, \sigma_{v_{0}}=0.04, \sigma_{v}=0.04\right)$} & \\
\hline 50 & 3 & $2.4 \mathrm{e}-2$ & $-2.1 \mathrm{e}-3$ & $-1.1 \mathrm{e}-2$ & $3.3 \mathrm{e}-2$ & $1.2 \mathrm{e}-2$ & $-2.1 \mathrm{e}-2$ & 2.7 & 2 & -1.5 \\
\hline 100 & 3 & $2.0 \mathrm{e}-$ & -5.8 & $-1.2 €$ & & & & & & -1.7 \\
\hline 500 & 3 & $3.2 \mathrm{e}-2$ & $-2.5 \mathrm{e}-3$ & $-1.3 \mathrm{e}-2$ & $1.9 \mathrm{e}-2$ & $6.3 \mathrm{e}-3$ & $-2.2 \mathrm{e}-2$ & $1.8 \mathrm{e}-2$ & $7.8 \mathrm{e}-3$ & $-1.8 \mathrm{e}-2$ \\
\hline 50 & 6 & $1.6 \mathrm{e}-2$ & $-2.8 \mathrm{e}-3$ & $-1.0 \mathrm{e}-2$ & $1.6 \mathrm{e}-2$ & $5.8 \mathrm{e}-3$ & $-2.3 e-2$ & $1.2 \mathrm{e}-2$ & $3.7 \mathrm{e}-3$ & $-1.9 \mathrm{e}-2$ \\
\hline 100 & 6 & $2.3 \mathrm{e}-2$ & -1 & $-1.1 \mathrm{e}$ & & 4.9 & -2 & 2 & 5.5 & $-1.9 \mathrm{e}-2$ \\
\hline 500 & 6 & $3.4 \mathrm{e}-2$ & $1.1 \mathrm{e}-3$ & $-1.2 \mathrm{e}-2$ & $1.3 \mathrm{e}-2$ & $4.6 \mathrm{e}-3$ & $-2.4 \mathrm{e}-2$ & $1.7 \mathrm{e}-2$ & $9.1 \mathrm{e}-3$ & $-2.0 \mathrm{e}-2$ \\
\hline 50 & 10 & $2.1 \mathrm{e}-$ & 4 & $-9.1 \mathrm{e}-$ & 3 & 60 & $-2.6 \mathrm{e}-2$ & $0_{0}$ & $3.7 \mathrm{e}-3$ & $-2.2 \mathrm{e}-2$ \\
\hline 100 & 10 & $2.1 \mathrm{e}-2$ & $-3.6 \mathrm{e}-4$ & $-1.1 \mathrm{e}$ & 3 & 1.2 & $-2.6 \mathrm{e}$ & 0.1 & & $-2.4 \mathrm{e}-2$ \\
\hline \multirow[t]{2}{*}{500} & 10 & $3.3 \mathrm{e}-2$ & $2.5 \mathrm{e}-3$ & $-1.1 \mathrm{e}-2$ & $7.5 \mathrm{e}-3$ & $9.1 \mathrm{e}-4$ & $-2.8 \mathrm{e}-2$ & 1.4 & $6.3 \mathrm{e}-3$ & $-2.5 \mathrm{e}-2$ \\
\hline & \multicolumn{9}{|c|}{$\mathrm{s} 12: \lambda_{0}=1, \lambda=5, \Lambda=1\left(\sigma_{u_{0}}=0.2 \sigma_{u}=0.2, \sigma_{v_{0}}=0.2, \sigma_{v}=0.04\right)$} & \\
\hline 50 & 3 & $1.2 \mathrm{e}-1$ & $2.8 \mathrm{e}-3$ & $-5.1 \mathrm{e}-2$ & $1.2 \mathrm{e}-1$ & $3.2 \mathrm{e}-2$ & $-3.8 \mathrm{e}-2$ & $1.5 \mathrm{e}-1$ & $4.7 \mathrm{e}-2$ & $-5.2 \mathrm{e}-2$ \\
\hline 100 & 3 & $1.3 \mathrm{e}-1$ & $1.8 \mathrm{e}-3$ & $-5.5 \mathrm{e}-2$ & $6.7 \mathrm{e}-2$ & $2.1 \mathrm{e}-2$ & $-4.2 \mathrm{e}-2$ & $1.1 \mathrm{e}-1$ & $3.2 \mathrm{e}-2$ & $-6.0 \mathrm{e}-2$ \\
\hline 500 & 3 & $1.6 \mathrm{e}-1$ & $6.9 \mathrm{e}-3$ & $-5.7 \mathrm{e}-2$ & $2.7 \mathrm{e}-2$ & $1.2 \mathrm{e}-2$ & $-4.4 \mathrm{e}-2$ & $1.0 \mathrm{e}-1$ & $2.8 \mathrm{e}-2$ & $-6.4 \mathrm{e}-2$ \\
\hline 50 & 6 & $1.3 \mathrm{e}$ & $2.4 \mathrm{e}$ & $-5.6 \mathrm{e}$ & 1 . & - & -3 & 7 & 9.1 & -6.5 \\
\hline 100 & 6 & $1.3 \mathrm{e}$ & & $-5.7 \epsilon$ & & -4.8 & & & & -6.7 \\
\hline 500 & 6 & $1.5 \mathrm{e}-1$ & $4.5 \mathrm{e}-3$ & $-6.0 \mathrm{e}-2$ & $2.6 \mathrm{e}-3$ & $-5.3 \mathrm{e}-3$ & $-3.8 \mathrm{e}-2$ & $7.7 \mathrm{e}-2$ & $8.4 \mathrm{e}-3$ & $-6.9 \mathrm{e}-2$ \\
\hline 50 & 10 & $1.2 \mathrm{e}-$ & $.5 \mathrm{e}-3$ & $-5.5 \mathrm{e}-2$ & $8.7 \mathrm{e}-3$ & $-1.3 \mathrm{e}-2$ & $-3.7 \mathrm{e}-2$ & $5.6 \mathrm{e}-$ & $1.7 \mathrm{e}-3$ & $-7.1 \mathrm{e}-2$ \\
\hline 100 & 10 & $1.3 \mathrm{e}-$ & $3.1 \mathrm{e}-3$ & $-5.8 \mathrm{e}-2$ & $-8.7 e-3$ & $-1.3 \mathrm{e}$ & $-3.8 \mathrm{e}-$ & $6.2 \mathrm{e}-2$ & $-1.1 \mathrm{e}-3$ & $-7.3 \mathrm{e}-2$ \\
\hline 500 & 10 & $1.5 \mathrm{e}-1$ & $2.9 \mathrm{e}-3$ & $-6.1 \mathrm{e}-2$ & $-7.8 \mathrm{e}-3$ & $-1.3 \mathrm{e}-2$ & $-3.9 \mathrm{e}-2$ & $6.9 \mathrm{e}-2$ & $5.3 \mathrm{e}-4$ & $-7.6 \mathrm{e}-2$ \\
\hline
\end{tabular}

When $\lambda_{0}=1$, the results for the 5th, median, and 95th percentiles are different. In case $\lambda=0.2$ (s5), the estimation of 5 th and 95 th percentiles of persistent technical efficiency are good as before (the 5 th percentile is overestimated, while the 95 th percentile is underestimated), but the relative deviation of median is even smaller, implying very good estimation of persistent technical efficiency in the middle of the distribution.

If $\lambda=1$ (s6), the median of both persistent and transient technical efficiency are estimated with a very small relative bias. As in scenario s5, the 5th percentile of persistent technical efficiency is overestimated, while the 95 th percentile is underestimated with a small relative error. Transient technical efficiency is estimated to be good for large values, but quite poorly for small values of technical efficiency.

When $\lambda=1$ and $\Lambda=1$ (s1 and s16), the median of both transient and persistent technical efficiency are estimated to be good to very good. In both s1 and s16 the 5th (95th) percentile of persistent technical efficiency are overestimated (underestimated) by more than the median. 
The same is true for transient technical efficiency. The GTRE estimator overestimates the 5th and underestimate the 95th percentile of transient technical efficiency. Similar to Table 4, the lowest levels of persistent technical efficiency are estimated in s16 slightly poorer than those in s1.

The case where $\lambda=1$ and $\Lambda=5$ (s11) is comparable to the case where $\lambda=1$ and $\Lambda=0.2$ (s6). However, now transient technical efficiency is estimated better than persistent technical efficiency. This is expected since other things being equal, small $\Lambda$ generally favors persistent technical efficiency, while large $\Lambda$ generally favors transient technical efficiency.

Finally, when $\lambda=5$ (s2 and s12), transient technical efficiency is estimated to be very good in the middle, and only slightly worse at low and high level of technical efficiency $(0.1 \%$ against $1 \%$ misestimation). In scenario s12 persistent technical efficiency is estimated to be good for the median and 95th percentile of technical efficiency, as it was the case with 'average' performance analysis.

Recall that in scenarios s2 and s12 relative bias has been increasing in $n$. This happens mainly because of the poor estimate of technical efficiency at the lowest percentile. At the lower end, technical efficiency is overestimated by more than it is underestimated at the upper end of the distribution. While relative mean deviation of the median and the 95th percentile is behaving as expected, it is increasing for the 5th percentiles of persistent technical efficiency. The mean squared deviations (not reported here) are decreasing as expected.

Table 10 presents the results when $\lambda_{0}=5$. In all four scenarios, s7, s8, s3, and s4, the median of persistent technical efficiency is predicted very accurately. The 5 th and 95 th percentiles are predicted with an error of only about $1-3 \%$. In scenario s4, where both $\lambda_{0}$ and $\lambda=5$, estimation of persistent technical efficiency is excellent.

Table 10: The Mean of the Relative Deviation Between the 5th, 50th and 95th Percentiles of the Estimated and True Technical Efficiency. $\sigma_{u_{0}}$ is high relative to $\sigma_{v_{0}}$, i.e., $\lambda_{0}=5$.

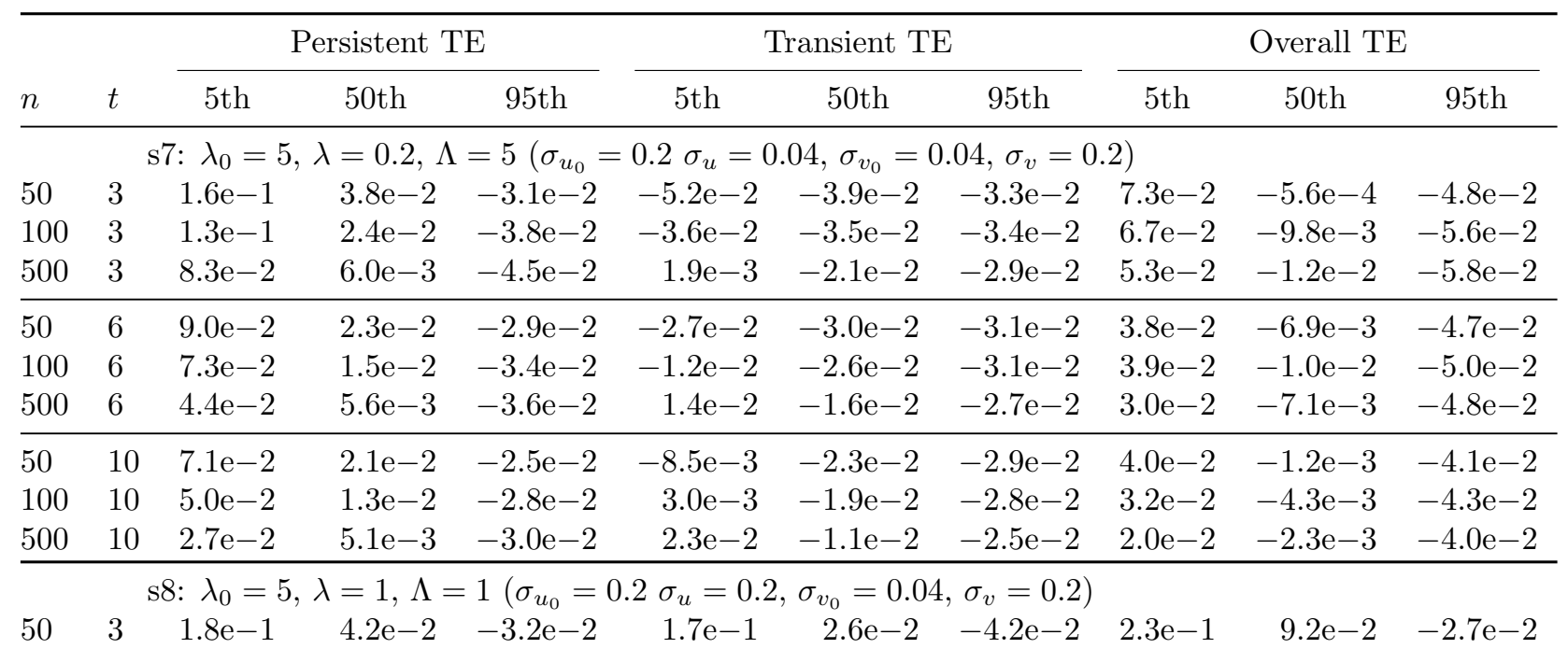

(continued on next page) 
Table 10 (Continued)

\begin{tabular}{|c|c|c|c|c|c|c|c|c|c|c|}
\hline \multirow[b]{2}{*}{$n$} & \multirow[b]{2}{*}{$t$} & \multicolumn{3}{|c|}{ Persistent TE } & \multicolumn{3}{|c|}{ Transient TE } & \multicolumn{3}{|c|}{ Overall TE } \\
\hline & & 5 th & 50 th & 95 th & 5 th & 50 th & 95th & 5 th & 50 th & 95th \\
\hline 100 & 3 & $1.6 \mathrm{e}-1$ & $3.0 \mathrm{e}-2$ & $-3.9 \mathrm{e}-2$ & $1.7 \mathrm{e}-1$ & $1.7 \mathrm{e}-2$ & $-4.9 \mathrm{e}-2$ & $2.0 \mathrm{e}-1$ & $6.9 \mathrm{e}-2$ & $-4.3 \mathrm{e}-2$ \\
\hline 500 & 3 & $1.1 \mathrm{e}-1$ & $7.2 \mathrm{e}-3$ & $-4.8 \mathrm{e}-2$ & $1.5 \mathrm{e}-1$ & $-6.6 e-4$ & $-6.3 e-2$ & $1.4 \mathrm{e}-1$ & $2.6 \mathrm{e}-2$ & $-6.9 \mathrm{e}-2$ \\
\hline 50 & 6 & $1.2 \mathrm{e}-1$ & $3.1 \mathrm{e}-2$ & $-3.0 \mathrm{e}-2$ & $1.7 \mathrm{e}-1$ & $2.1 \mathrm{e}-2$ & $-4.9 \mathrm{e}-2$ & $1.8 \mathrm{e}-1$ & $7.1 \mathrm{e}-2$ & $-3.8 \mathrm{e}-2$ \\
\hline 100 & 6 & $9.9 \mathrm{e}-2$ & $1.9 \mathrm{e}-2$ & $-3.5 \mathrm{e}-2$ & $1.7 e-1$ & $1.3 \mathrm{e}-2$ & $-5.4 \mathrm{e}-2$ & $1.6 \mathrm{e}-1$ & $5.0 \mathrm{e}-2$ & $-5.1 \mathrm{e}-2$ \\
\hline 500 & 6 & $5.7 \mathrm{e}-2$ & $5.3 e-3$ & $-3.9 \mathrm{e}-2$ & $1.4 \mathrm{e}-1$ & $-4.5 \mathrm{e}-3$ & $-6.6 \mathrm{e}-2$ & $1.0 \mathrm{e}-1$ & $1.5 \mathrm{e}-2$ & $-7.0 \mathrm{e}-2$ \\
\hline 50 & 10 & $8.1 \mathrm{e}-2$ & $2.4 \mathrm{e}-2$ & $-2.7 \mathrm{e}-2$ & $1.7 \mathrm{e}-1$ & $1.5 \mathrm{e}-2$ & $-5.4 \mathrm{e}$ & $1.5 \mathrm{e}$ & $5.4 \mathrm{e}-2$ & $-4.4 \mathrm{e}-2$ \\
\hline 100 & 10 & $5.9 \mathrm{e}-2$ & $1.5 \mathrm{e}-2$ & $-3.1 \mathrm{e}-2$ & $1.5 \mathrm{e}-1$ & $4.6 \mathrm{e}-3$ & $-6.0 \mathrm{e}-2$ & $1.2 \mathrm{e}-1$ & $3.2 \mathrm{e}-2$ & $-5.7 \mathrm{e}-2$ \\
\hline \multirow[t]{2}{*}{500} & 10 & $3.9 \mathrm{e}-2$ & $6.8 \mathrm{e}-3$ & $-3.3 \mathrm{e}-2$ & $1.3 \mathrm{e}-1$ & $-6.2 \mathrm{e}-3$ & $-6.7 \mathrm{e}-2$ & $9.2 \mathrm{e}-2$ & $1.1 \mathrm{e}-2$ & $-6.8 \mathrm{e}-2$ \\
\hline & \multicolumn{9}{|c|}{$\mathrm{s} 3: \lambda_{0}=5, \lambda=1, \Lambda=5\left(\sigma_{u_{0}}=0.2 \sigma_{u}=0.04, \sigma_{v_{0}}=0.04, \sigma_{v}=0.04\right)$} & \\
\hline 50 & 3 & $2.2 \mathrm{e}-2$ & $7.4 \mathrm{e}-3$ & $-1.5 \mathrm{e}-2$ & $3.2 \mathrm{e}-2$ & $5.9 \mathrm{e}-3$ & $-8.8 \mathrm{e}-3$ & $2.8 \mathrm{e}-2$ & $1.5 \mathrm{e}-2$ & $-9.1 \mathrm{e}-3$ \\
\hline 100 & 3 & $1.5 \mathrm{e}-2$ & $4.2 \mathrm{e}-3$ & $-1.8 \mathrm{e}-2$ & $3.2 \mathrm{e}-2$ & $4.4 \mathrm{e}-3$ & $-1.0 \mathrm{e}-2$ & $2.4 \mathrm{e}-2$ & $1.1 \mathrm{e}-2$ & $-1.3 \mathrm{e}-2$ \\
\hline 500 & 3 & $6.4 \mathrm{e}-3$ & $1.1 \mathrm{e}-4$ & $-1.9 \mathrm{e}-2$ & $3.0 \mathrm{e}-2$ & $8.3 e-4$ & $-1.3 \mathrm{e}-2$ & $1.1 \mathrm{e}-2$ & $3.9 \mathrm{e}-3$ & $-1.8 \mathrm{e}-2$ \\
\hline 50 & 6 & $1.6 \mathrm{e}-2$ & $5.7 \mathrm{e}-3$ & $-1.4 \mathrm{e}-2$ & $3.2 \mathrm{e}-2$ & $3.9 \mathrm{e}-3$ & $-1.0 \mathrm{e}-2$ & $2.3 \mathrm{e}-2$ & $1.3 \mathrm{e}-2$ & $-1.1 \mathrm{e}-2$ \\
\hline 100 & 6 & $1.3 \mathrm{e}-2$ & $3.8 \mathrm{e}-3$ & $-1.6 \mathrm{e}-2$ & $2.9 \mathrm{e}-2$ & $1.5 \mathrm{e}-3$ & $-1.2 \mathrm{e}-2$ & $2.0 \mathrm{e}-2$ & $8.0 \mathrm{e}-3$ & $-1.6 \mathrm{e}-2$ \\
\hline 500 & 6 & $2.8 \mathrm{e}-3$ & $-2.3 \mathrm{e}-3$ & $-1.8 \mathrm{e}-2$ & $2.6 \mathrm{e}-2$ & $-1.2 \mathrm{e}-3$ & $-1.4 \mathrm{e}-2$ & $5.7 \mathrm{e}-3$ & $-3.9 \mathrm{e}-4$ & $-2.0 \mathrm{e}-2$ \\
\hline 50 & 10 & $1.5 \mathrm{e}-2$ & $7.3 e-3$ & $-1.4 \mathrm{e}-2$ & $3.0 \mathrm{e}-2$ & $2.2 \mathrm{e}-3$ & $-1.2 \mathrm{e}-2$ & $2.1 \mathrm{e}-2$ & $1.2 \mathrm{e}-2$ & $-1.4 \mathrm{e}-2$ \\
\hline 100 & 10 & $1.1 \mathrm{e}-2$ & $3.6 \mathrm{e}-3$ & $-1.6 \mathrm{e}-2$ & $2.8 \mathrm{e}-2$ & $7.3 e-4$ & $-1.3 e-2$ & $1.7 \mathrm{e}-2$ & $7.5 e-3$ & $-1.7 \mathrm{e}-2$ \\
\hline \multirow[t]{2}{*}{500} & 10 & $3.7 \mathrm{e}-3$ & $-1.5 \mathrm{e}-3$ & $-1.8 \mathrm{e}-2$ & $2.4 \mathrm{e}-2$ & $-2.1 \mathrm{e}-3$ & $-1.4 \mathrm{e}-2$ & $5.8 \mathrm{e}-3$ & $-8.2 \mathrm{e}-4$ & $-2.2 \mathrm{e}-2$ \\
\hline & \multicolumn{9}{|c|}{$\mathrm{s} 4: \lambda_{0}=5, \lambda=5, \Lambda=1\left(\sigma_{u_{0}}=0.2 \sigma_{u}=0.2, \sigma_{v_{0}}=0.04, \sigma_{v}=0.04\right)$} & \\
\hline 50 & 3 & $6.0 \mathrm{e}-2$ & $1.4 \mathrm{e}-2$ & $-2.0 \mathrm{e}-2$ & $6.6 \mathrm{e}-2$ & $2.0 \mathrm{e}-2$ & $-3.2 \mathrm{e}-2$ & $6.8 \mathrm{e}-2$ & $3.7 \mathrm{e}-2$ & $-1.3 \mathrm{e}-2$ \\
\hline 100 & 3 & $4.3 \mathrm{e}-2$ & $6.8 \mathrm{e}-3$ & $-2.1 \mathrm{e}-2$ & $4.2 \mathrm{e}-2$ & $1.4 \mathrm{e}-2$ & $-3.3 \mathrm{e}-2$ & $3.9 \mathrm{e}-2$ & $2.0 \mathrm{e}-2$ & $-1.6 \mathrm{e}-2$ \\
\hline 500 & 3 & $2.6 \mathrm{e}-2$ & $-1.7 \mathrm{e}-3$ & $-2.1 \mathrm{e}-2$ & $2.8 \mathrm{e}-2$ & $1.1 \mathrm{e}-2$ & $-3.4 \mathrm{e}-2$ & $1.7 \mathrm{e}-2$ & $5.5 e-3$ & $-1.8 \mathrm{e}-2$ \\
\hline 50 & 6 & $3.5 \mathrm{e}-2$ & $1.4 \mathrm{e}-2$ & $-1.3 \mathrm{e}-2$ & $1.3 \mathrm{e}-2$ & $3.8 \mathrm{e}-3$ & $-2.8 \mathrm{e}-2$ & $2.6 \mathrm{e}-2$ & $1.3 \mathrm{e}-2$ & $-1.7 \mathrm{e}-2$ \\
\hline 100 & 6 & $2.5 \mathrm{e}-2$ & $8.2 \mathrm{e}-3$ & $-1.5 \mathrm{e}-2$ & $1.0 \mathrm{e}-2$ & $3.3 \mathrm{e}-3$ & $-2.7 e-2$ & $1.7 \mathrm{e}-2$ & $6.0 \mathrm{e}-3$ & $-1.9 \mathrm{e}-2$ \\
\hline 500 & 6 & $1.5 \mathrm{e}-2$ & $3.3 \mathrm{e}-3$ & $-2.1 \mathrm{e}-2$ & $1.5 \mathrm{e}-2$ & $5.4 \mathrm{e}-3$ & $-2.4 \mathrm{e}-2$ & $1.3 \mathrm{e}-2$ & $2.5 \mathrm{e}-3$ & $-1.9 \mathrm{e}-2$ \\
\hline 50 & 10 & $2.9 \mathrm{e}-2$ & $1.7 \mathrm{e}-2$ & $-6.0 \mathrm{e}-3$ & $-1.3 e-2$ & $-6.0 \mathrm{e}-3$ & $-2.7 \mathrm{e}-2$ & $1.4 \mathrm{e}-2$ & $4.8 \mathrm{e}-3$ & $-2.1 \mathrm{e}-2$ \\
\hline 100 & 10 & $2.2 \mathrm{e}-2$ & $1.2 \mathrm{e}-2$ & $-1.1 \mathrm{e}-2$ & $-6.9 \mathrm{e}-3$ & $-3.9 \mathrm{e}-3$ & $-2.6 \mathrm{e}-2$ & $1.3 \mathrm{e}-2$ & $1.2 \mathrm{e}-3$ & $-2.4 \mathrm{e}-2$ \\
\hline 500 & 10 & $1.1 \mathrm{e}-2$ & $5.6 \mathrm{e}-3$ & $-1.9 \mathrm{e}-2$ & $2.6 \mathrm{e}-3$ & $1.6 \mathrm{e}-3$ & $-2.2 \mathrm{e}-2$ & $9.7 \mathrm{e}-3$ & $-2.5 \mathrm{e}-4$ & $-2.3 \mathrm{e}-2$ \\
\hline
\end{tabular}

The median of transient technical efficiency is estimated to be good to very good in all scenarios with only a small error. When $\lambda=\Lambda=1$ (s8), technical efficiency at the lower end is estimated very poorly, while at the higher end it is estimated with a relative bias of about $10 \%$. When $\lambda=1, \Lambda=5$ (s3), the 5th and 95th percentiles are over- and underestimated, but the misestimation is rather small. Finally, in the most favorable scenario where $\lambda=5, \Lambda=5$ (s4), both the 5th percentile and the median are estimated very accurately and are overestimated, except for very large samples, where they are underestimated, while 95th percentile is estimated less precisely and is underestimated throughout.

In sum, Tables 8-10 reveal several very interesting features of efficiency estimators in the GTRE model. First, the biases of lower and higher levels of technical efficiency estimates have mostly opposite signs. For a practitioner this means that by just knowing the direction of bias of the estimator on 'average' might not be informative when the interest lies in estimating the 
benchmark firms or the least efficient firms. Second, even if the technical efficiency is estimated accurately in the middle, there is good chance that lower and higher levels of technical efficiency are estimated quite poorly. Third, in most scenarios the estimator becomes good for all levels of technical efficiency when $n t$ is quite large. We illustrate how to make use of these tables in the next section when we report results based on several real data sets.

\section{Empirical Applications}

We have shown the performance of the estimator in Monte Carlo simulations, but it is also worthwhile to show how the estimator works in practice. Here we consider four separate data sets used in empirical papers which correspond to different scenarios based on the estimated values of $\lambda_{0}, \lambda$, and $\Lambda$. We report the estimated parameters $\hat{\lambda}_{0}, \hat{\lambda}, \hat{\Lambda}, \hat{\sigma}_{u_{0}}, \hat{\sigma}_{u}, \hat{\sigma}_{v_{0}}$ and $\hat{\sigma}_{v}{ }^{7}$ the description of the panel data structure in Table $11^{8}$ as well as the summary statistics of the technical or cost efficiencies in Table 12. In practice, we would not get the estimated values of lambdas to be equal to $0.2,1$, and 5 which we used in the simulation study. If we roughly consider the value above 2 as 5 (well above unity), value below 0.5 as 0.2 (well below unity), and the values between 0.5 and 2 as 1 , we can crudely classify the empirical results into scenarios that we have seen in the MC study.

\subsection{Swiss Railway Data}

These data come from Filippini and Greene (2016), who estimate a cost function for 50 Swiss railroads over the period 1985-1997. They model total costs as a function of input prices (labor, capital and energy), outputs (numbers of passenger-kilometers and freight ton-kilometers), the length of network, and the number of stops. They also include time dummies to account for technological progress. Here we estimate exactly the same model. The total costs and prices of labor and capital are normalized by price of energy to linear homogeneity (in input prices) property of the cost function.

Railroads are observed for 12.1 years on average, which approximates the setup where $n=50$ and $t=10$. The estimated parameters of interest in Table 11 (and their standard errors in parentheses) are $\hat{\lambda}_{0}=3.6(0.22), \hat{\lambda}=1.5(0.39)$, and $\hat{\Lambda}=7.2(1.1)$. These put us into scenario s3. Table 11 presents the summary statistics of the estimated cost efficiency scores. Persistent cost efficiency are estimated to be good on 'average' while estimation of transient cost efficiency is only fair. Further, Table 10 suggests that persistent cost efficiency (first row in Table 12) is overestimated by $1.5 \%$ at the lower end, overestimated by $0.7 \%$ in the middle, and underestimated by about $1.4 \%$ at the upper end of the cost efficiency distribution. The signs of misestimation of transient (second row) and overall (third row) cost efficiency at different portions of distribution

\footnotetext{
${ }^{7}$ Note that $\lambda_{0}=\sigma_{u_{0}} / \sigma_{v_{0}}$, and $\Lambda=\sigma_{u_{0}} / \sigma_{u}=\sqrt{1+1 / \lambda^{2}} \sigma_{u_{0}} / \sigma$. The standard errors are obtained using Delta method.

${ }^{8}$ Since our interest lies in estimation of technical efficiencies, the parameters of the respective production or cost function are not reported to conserve space. They are available from authors upon request.
} 
Table 11: Parameter Estimates

\begin{tabular}{lllll}
\hline Parameter & Data1 $^{1}$ & Data2 $^{2}$ & Data3 $^{3}$ & Data4 $^{4}$ \\
\hline$\hat{\lambda}_{0}$ & $3.6(0.22)$ & $1.8(0.28)$ & $0.93(0.05)$ & $0.0019(1.4)$ \\
$\hat{\lambda}$ & $1.5(0.39)$ & $1.3(0.21)$ & $2.3(0.32)$ & $0.82(0.45)$ \\
$\hat{\Lambda}$ & $7.2(1.1)$ & $1.9(0.26)$ & $3.4(0.32)$ & $0.00092(0.67)$ \\
$\hat{\sigma}_{u_{0}}$ & $0.56(0.015)$ & $0.15(0.012)$ & $0.36(0.021)$ & $0.00021(0.16)$ \\
$\hat{\sigma}_{u}$ & $0.078(0.012)$ & $0.078(0.0085)$ & $0.11(0.0064)$ & $0.23(0.1)$ \\
$\hat{\sigma}_{v_{0}}$ & $0.16(0.01)$ & $0.083(0.0086)$ & $0.39(0.012)$ & $0.11(0.015)$ \\
$\hat{\sigma}_{v}$ & $0.052(0.0059)$ & $0.062(0.0038)$ & $0.046(0.0039)$ & $0.28(0.032)$ \\
$\bar{T}_{i}$ & 12.1 & 6 & 12.99 & 6 \\
$N$ & 50 & 247 & 82 & 171 \\
$N T$ & 605 & 1482 & 1065 & 1026 \\
\hline
\end{tabular}

Standard errors in parentheses;

${ }^{1}$ Swiss Railway data, 1985-1997;

2 Spanish dairy farms, 1993-1998;

${ }^{3}$ US Fossil-fuel-fired steam electric power-generating plants, 1986-1999;

${ }^{4}$ Indonesian rice farms, 1971-1986

Table 12: Summary Statistics for Different Types of Cost/Technical Efficiency

\begin{tabular}{llllllll}
\hline & & Min & $5 \%$ & Mean & $50 \%$ & $95 \%$ & Max \\
\hline \multirow{2}{*}{ Data1 } & Persistent & 0.18 & 0.34 & 0.64 & 0.64 & 0.90 & 0.93 \\
& Residual & 0.74 & 0.88 & 0.94 & 0.95 & 0.97 & 0.98 \\
\multirow{5}{*}{ Data2 } & Overall & 0.17 & 0.32 & 0.60 & 0.60 & 0.85 & 0.90 \\
& Persistent & 0.69 & 0.77 & 0.89 & 0.91 & 0.95 & 0.97 \\
& Residual & 0.73 & 0.89 & 0.94 & 0.95 & 0.97 & 0.98 \\
& Overall & 0.57 & 0.71 & 0.84 & 0.85 & 0.91 & 0.95 \\
Data3 & Persistent & 0.62 & 0.67 & 0.77 & 0.78 & 0.86 & 0.89 \\
& Residual & 0.58 & 0.83 & 0.91 & 0.93 & 0.96 & 0.99 \\
& Overall & 0.42 & 0.59 & 0.71 & 0.72 & 0.81 & 0.86 \\
Data4 & Persistent & 1.00 & 1.00 & 1.00 & 1.00 & 1.00 & 1.00 \\
& Residual & 0.63 & 0.75 & 0.84 & 0.85 & 0.90 & 0.95 \\
& Overall & 0.63 & 0.75 & 0.84 & 0.85 & 0.90 & 0.95 \\
\hline
\end{tabular}

are the same as those of persistent cost efficiency, the magnitudes are twice as large for lower levels and about the same for higher levels of cost efficiency.

\subsection{Dairy Farms Data}

This data set is on Spanish dairy farms (Alvarez et al. 2004). Output produced (milk in liters) is specified as a function of number of milking cows, size of the land devoted to pasture and crops (in hectares), labor (man-equivalent units), feeds (in tons), time trend, and time trend 
squared. The data are balanced panel with $t=6$ years (1993-1998) and $n=247$ farms, which is crudely $n=500$ and $t=6$ in our simulation study.

The estimate of $\lambda$ is equal $1.26(0.21)$ is roughly unity. The estimates of $\lambda_{0}$ and $\Lambda$ are $1.79(0.28)$ and $1.902(0.26)$. We cannot clearly classify this case in terms of lambdas from simulation study, but if we consider 1.79 closer to unity and 1.902 closer to 5 , this puts us into scenario s11. ${ }^{9}$ Recall that in this scenario all types of technical efficiency are estimated to be fair at best considering the three criteria we used. The transient technical efficiency estimates can only be trusted in the middle of the distribution, where they are underestimated by only $0.02 \%$, while other types of technical efficiency are underestimated by about $1 \%$. Given the results of the Monte-Carlo study, it would be difficult to put any faith in the technical efficiency estimates for Spanish dairy farms at the low and upper end of persistent and overall technical efficiency distribution, where they are misestimated by $6-12 \%$.

\subsection{US Electric Utilities Data}

These data come from Kumbhakar and Tsionas (2011), who use data 82 fossil-fuel-fired steam electric power-generating plants in the USA over the period 1986-1999 (case corresponds to $n=100$ and $t=10$ ). Here we estimate a cost function that is specified as a function of output (net steam electric power generation in megawatt-hours), prices of labor, fuel, capital, and time trend.

The first thing to notice in Table 11 is that estimates $\hat{\lambda}_{0}=0.93(0.05), \hat{\lambda}=2.29(0.32)$, and $\hat{\Lambda}=3.43(0.32)$ do not put us directly into any of the scenarios in our simulations. This constellation of lambdas resembles that of scenario s12, where $\lambda_{0}=1, \lambda=5$, and $\Lambda=1$, except that $\hat{\Lambda}$ is about 5 . We call this scenario s12' since Table 6 suggests that this would be the next scenario after $\mathrm{s} 2$ and $\mathrm{s} 12$, and the estimation would supposedly possess the same properties, namely that persistent cost efficiency estimates are fair, whereas transient cost efficiency are reliable. Indeed, according to scenario s12 in Table 9 for s12, average and large transient cost efficiency are underestimated by about $1.5 \%$, whereas the small transient cost efficiency are underestimated by only $0.8 \%$. Conversely, the low persistent cost efficiency are overestimated by staggering $13 \%$, while persistent cost efficiency are estimated quite precisely in the middle. Taking into account all criteria that we reported in the MC study, it would be difficult to trust the estimated persistent cost efficiency.

\subsection{Indonesian Rice Farm Data}

The data on 171 Indonesian Rice Farms for 6 growing seasons come from Horrace and Schmidt (2000). The authors specify the production of output (rough rice) in kilogram as a function of seed in kilogram, urea in kilogram, total labor (excluding harvest labor), total area that farmers

\footnotetext{
${ }^{9}$ If we consider all lambdas being closer to unity, this puts us into s16, where the goodness of GTRE estimator according to Tables 4 and 6 is similar to that of s11.
} 
Table 13: Likelihood Values and Likelihood-Ratio Tests

\begin{tabular}{|c|c|c|c|c|c|c|c|c|c|c|c|}
\hline \multirow[t]{2}{*}{ Model } & \multicolumn{3}{|c|}{ Parameters $^{\mathrm{a}}$} & \multicolumn{2}{|c|}{ data 1} & \multicolumn{2}{|c|}{ data 2} & \multicolumn{2}{|c|}{ data 3} & \multicolumn{2}{|c|}{ data 4} \\
\hline & $\sigma_{v_{0}}$ & $\sigma_{u_{0}}$ & $\sigma_{u}$ & $\log L$ & $L R^{\mathrm{b}}$ & $\log L$ & $L R^{\mathrm{b}}$ & $\log L$ & $L R^{\mathrm{b}}$ & $\log L$ & $L R^{\mathrm{b}}$ \\
\hline M1 & 1 & 1 & 1 & 603.06 & & 1346.01 & & 982.81 & & -322.40 & \\
\hline M2 & 1 & 1 & 0 & 595.87 & 14.38 & 1340.45 & 11.11 & 955.01 & 55.60 & -322.67 & 0.55 \\
\hline M3 & 1 & 0 & 1 & 600.70 & 4.71 & 1340.13 & 11.74 & 982.67 & 0.27 & -322.40 & 0.00 \\
\hline M4 & 0 & 1 & 1 & 602.19 & 1.74 & 1324.47 & 43.08 & 953.37 & 58.89 & -327.76 & 10.74 \\
\hline M5 & 0 & 1 & 0 & 596.39 & 13.34 & 1323.44 & 45.12 & 930.23 & 105.15 & -327.76 & 10.74 \\
\hline M6 & 0 & 0 & 1 & -116.06 & 1438.24 & 813.59 & 1064.83 & 67.73 & 1830.16 & -333.59 & 22.39 \\
\hline
\end{tabular}

a Zero means that the respective variance parameter is restricted to zero;

${ }^{\mathrm{b}} L R=-2\left(\log L-\log L^{\mathrm{GTRE}}\right)$.

cultivated with rice, measured in hectares, and three dummy variables representing the varieties, use of pesticide/herbicide, time trend, and time trend squared.

For this data the results in Table 11 suggests that estimates $\hat{\lambda}_{0}$ and $\hat{\Lambda}$ are statistically insignificant since the estimated standard errors are quite big. This implies that that persistent technical inefficiency is absent. Unlike before, the estimated $\Lambda$ is close to one, putting the results in s14, where $\lambda_{0}=0.2, \lambda=1$, and $\Lambda=0.2$. This is worse than s10, because according to the summary in Table 6 even transient technical efficiency estimates are not reliable. Thus, the estimator does not estimate any type of technical efficiency reliably for any observation for the Indonesian rice farms.

Interestingly, the results suggest that persistent technical inefficiency is absent. Transient technical inefficiency definitely exists, but it cannot be reliably estimated. Transient technical inefficiency is quite large for some farms. These findings maybe due to the fact that rice production has a very long tradition/history. Consequently, persistent technical inefficiency has been eliminated, while short-run technical inefficiency is present due to possibly weather and/or climate related conditions.

\subsection{Appropriateness of the GTRE Model in the applications}

The GTRE model provides estimates of two types of inefficiency while taking firm-effects and noise into account. From a practitioner's point of view the issue is whether such a model is consistent with the data. Our goal therefore is to check the performance of the GTRE model to the models where one or more of the error components (their variances) are restricted to zero.

Table 13 provides the values of log-likelihood function for different combination of restrictions put on the variance parameters. This gave us six different models. First three columns describe restrictions imposed on the model. A value of unity means the corresponding variance parameter is free and a value of zero means the parameter is restricted to zero. First three mod- 
els (M1, M2 and M3) include random firm-effects (heterogeneity). M1 corresponds to GTRE model where none of the variance parameter is restricted to zero. M2 does not include transient technical inefficiency, while M3 does not include persistent technical inefficiency. Models M4, M5, and M6 exclude firm-heterogeneity. Model 4 considers both persistent and transient persistent technical efficiencybut firm-effects are excluded. Thus in comparison to M1, M2, M3, M4 restricts parameter (firm-effects component) to zero. M5 accounts only for persistent technical inefficiency, while M6 accounts only for transient technical inefficiency. M5 and M6 restrict two variance parameters (error components) to zero. For each data set, the second column shows the LR statistic that can be used to test appropriateness of the GTRE model. Given that the zero restrictions are on the boundary of the parameter values, the LR statistics has a mixed $\chi^{2}$ distribution (see Coelli 1995). The critical values of the mixed $\chi^{2}$ distribution can be found in Kodde and Palm (1986). With 2 degrees of freedom the critical values from the mixed $\chi^{2}$ distribution are 8.273 and 5.138 for significance levels 0.01 and 0.05 , respectively. The critical values of the mixed $\chi^{2}$ distribution with 1 degree of freedom for significance levels 0.01 and 0.05 are 5.412 and 2.705, respectively. Models with two restrictions (M5 and M6) are rejected at the $1 \%$ level in all data sets.

Statistical significance of restriction on a single variance parameter differs across data sets. Table 13 suggests that for data 1, it is important to account for both types of cost inefficiency, but not random firm heterogeneity (Kumbhakar and Heshmati (1995) type of the model). If one accounts for random firm heterogeneity, then at least transient technical inefficiency needs to be modeled (4.71 is only statistically significant at $5 \%$ ). For data set 2 , none of the restriction is justified, so that GTRE model is appropriate. For data set 3, both GTRE and TRE models that account for firm heterogeneity and transient technical inefficiency are appropriate. As long as heterogeneity and one of two types of technical inefficiency is modeled for data set 4, the GTRE does not seem any better.

In sum, Table 13 suggests that the GTRE model is not always the best (most appropriate) model for any data set. Some more parsimonious model might also be consistent with the data.

\section{Conclusion}

In this paper we consider estimation of both persistent and time-varying (long-run and shortrun/transient) inefficiency in a panel data model that also allows for random firm-effects (heterogeneity) and noise. In order to do so we had to make distributional assumption on each of the error components which are assumed to be random. We used simulated maximum likelihood approach to estimate parameters of the model, and then used the expected values of conditional means of the efficiency components conditional on the residuals to predict persistent and transient efficiency components. Given that the model is complex and relatively new, our main goal is to address three main concerns. The first concern is whether the model can accurately identify all the four components, and if so how precisely can the model estimate them? Second, there are 
two time-varying random components (time-varying inefficiency and noise), and the concern is whether the model can separate noise from transient inefficiency, and if so how precisely can the model estimate transient inefficiency? Third, how well are persistent and transient inefficiency estimated under different scenarios, viz., under different configurations of the variances of the four random components?

We address these concerns in terms of a series of simulated models. We found that the goodness of the technical efficiency estimates hinges on how the variance parameters of the four error components are related. Two comments are worth emphasizing. First, except for the case where both random effects and noise are virtually nonexistent, the estimator can provide reliable technical efficiency estimates of at most one type of technical efficiency. The other type, or in some scenarios, both types are estimated so poorly that we do not recommend performing efficiency analysis. Second, even if technical efficiency estimates can be trusted in the middle part of the distribution, very often the smallest and the largest technical efficiencies (which are at the ends of the distribution) are estimated quite imprecisely.

We have also evaluated the performance of the estimator using real data. We could not confirm a single case where both types of technical efficiency are estimated reliably from the statistical point of view. For most data sets, only one type of technical efficiency estimates can be trusted (statistically). In one case, the method cannot be trusted for estimating any type of technical efficiency. We also show that the GTRE model does not uniformly and overwhelmingly outperform simpler models.

Our hope is that the information that provided here will be useful to the practitioners. We do not discourage performing efficiency analysis altogether, but we rather suggest treating the obtained estimates with care since failure to do so might lead to technical efficiency estimates that are in essence useless.

\section{References}

Aigner, D., Lovell, C. K. and Schmidt, P.: 1977, Formulation and estimation of stochastic frontier production function models, Journal of Econometrics 6(1), 21-37.

Alvarez, A., Arias, C. and Greene, W. H.: 2004, Accounting for Unobservables in Production Models: Management and Inefficiency. Unpublished manuscript.

Battese, G. E. and Coelli, T. J.: 1988, Prediction of firm-level technical efficiencies with a generalized frontier production function and panel data, Journal of Econometrics 38(3), 387399.

Battese, G. E. and Coelli, T. J.: 1992, Frontier production functions, technical efficiency and panel data: With application to paddy farmers in India, Journal of Productivity Analysis 3(1-2), 153-169. 
Bos, J., Koetter, M., Kolari, J. and Kool, C.: 2009, Effects of heterogeneity on bank efficiency scores, European Journal of Operational Research 195(1), 251-261.

URL: http://dx.doi.org/10.1016/j.ejor.2008.01.019

Butler, J. S. and Moffitt, R.: 1982, A Computationally Efficient Quadrature Procedure for the One-Factor Multinomial Probit Model, Econometrica 50(3), 761-764.

Coelli, T. J.: 1995, Estimators and Hypothesis Tests for a Stochastic Frontier Function: A Monte Carlo Analysis, Journal of Productivity Analysis 6(3), 247-268.

Colombi, R., Kumbhakar, S. C., Martini, G. and Vittadini, G.: 2014, Closed-skew normality in stochastic frontiers with individual effects and long/short-run efficiency, Journal of Productivity Analysis 42(2), 123-136.

Cornwell, C., Schmidt, P. and Sickles, R. C.: 1990, Production frontiers with cross-sectional and time-series variation in efficiencylevels, Journal of Econometrics 46, 185-200.

Filippini, M. and Greene, W. H.: 2016, Persistent and Transient Productive Inefficiency: A Maximum Simulated Likelihood Approach, Journal of Productivity Analysis 45(2), 187-196.

Greene, H. W.: 2005, Reconsidering heterogeneity in panel data estimators of the stochastic frontier model, Journal of Econometrics 126(2), 269-303.

Horrace, W. C. and Schmidt, P.: 2000, Multiple comparisons with the best, with economic applications, Journal of Applied Econometrics 15(1), 1-26.

Jondrow, J., Knox Lovell, C. A., Materov, I. S. and Schmidt, P.: 1982, On the estimation of technical inefficiency in the stochastic frontier production function model, Journal of Econometrics 19(2-3), 233-238.

Kodde, D. A. and Palm, F. C.: 1986, Wald Criteria for Jointly Testing Equality and Inequality Restrictions, Econometrica 54(5), 1243-48.

Kumbhakar, S. C.: 1987, The specification of technical and allocative inefficiency in stochastic production and profit frontiers, Journal of Econometrics 34(3), 335-348.

Kumbhakar, S. C.: 1990, Production frontiers, panel data, and time-varying technical inefficiency, Journal of Econometrics 46(1-2), 201-211.

Kumbhakar, S. C.: 1991, The measurement and decomposition of cost-inefficiency: The translog cost system, Oxford Economic Papers, New Series 43(4), 667-683.

Kumbhakar, S. C. and Heshmati, A.: 1995, Efficiency Measurement in Swedish Dairy Farms: An Application of Rotating Panel Data, American Journal of Agricultural Economics 77(3), 660674. 
Kumbhakar, S. C. and Hjalmarsson, L.: 1993, Technical efficiency and technical progress in swedish dairy farms, in H. O. Fried, C. K. Lovell and S. S. Schmidt (eds), The Measurement of Productive Efficiency. Techniques and Applications, Oxford University Press, Oxford, pp. 256270.

Kumbhakar, S. C. and Hjalmarsson, L.: 1995, Labour-Use Efficiency in Swedish Social Insurance Offices, Journal of Applied Econometrics 10(1), 33-47.

Kumbhakar, S. C., Lien, G. and Hardaker, J. B.: 2014, Technical efficiency in competing panel data models: a study of Norwegian grain farming, Journal of Productivity Analysis 41(2), 321337.

Kumbhakar, S. C. and Tsionas, E. G.: 2011, Stochastic error specification in primal and dual production systems, Journal of Applied Econometrics 26(2), 270-297.

Kumbhakar, S. C. and Wang, H.-J.: 2005, Estimation of growth convergence using a stochastic production frontier approach, Economics Letters 88(3), 300-305.

Lee, Y. H.: 2010, Group-specific stochastic production frontier models with parametric specifications, European Journal of Operational Research 200(2), 508-517.

URL: http://dx.doi.org/10.1016/j.ejor.2009.01.030

Meeusen, W. and van den Broeck, J.: 1977, Efficiency estimation from Cobb-Douglas production functions with composed error, International Economic Review 18(2), 435-444.

Mester, L. J.: 1997, Measuring efficiency at U.S. banks: Accounting for heterogeneity is important, European Journal of Operational Research 98(2), 230-242.

URL: http://dx.doi.org/10.1016/S0377-2217(96)00344-X

Mundlak, Y.: 1961, Empirical Production Function Free of Management Bias, Journal of Farm Economics 43(1), 44-56.

Pitt, M. M. and Lee, L.-F.: 1981, The measurement and sources of technical inefficiency in the Indonesian weaving industry, Journal of Development Economics 9(1), 43-64.

Schmidt, P. and Sickles, R. C.: 1984, Production frontier and panel data, Journal of Business and Economic Statistics 2(4), 367-374.

Tsionas, E. G. and Kumbhakar, S. C.: 2014, Firm Heterogeneity, Persistent and Transient Technical Inefficiency: a Generalized True Random-Effects Model, Journal of Applied Econometrics 29(1), 110-132.

Yip, T. L., Sun, X. Y. and Liu, J. J.: 2011, Group and individual heterogeneity in a stochastic frontier model: Container terminal operators, European Journal of Operational Research 213(3), 517-525.

URL: http://dx.doi.org/10.1016/j.ejor.2011.03.040 